\title{
The Lysianassoid Amphipod Genera Lepidepecreoides and Lepidepecreum in Southern Waters (Crustacea: Lysianassidae: Tryphosinae)
}

\author{
J.K. LOWRY AND H.E. STODDART \\ Division of Invertebrate Zoology, \\ Australian Museum, 6 College Street, Sydney NSW 2010, Australia \\ jimlowry@crustacea.net · helenst@austmus.gov.au
}

\begin{abstract}
This paper reports on the first records of the lysianassid amphipod genera Lepidepecreoides and Lepidepecreum from Australia and Chile. In Lepidepecreoides two new species are described from Australian waters, one new species is described from Chilean waters, a new species is designated for the record (Griffiths, 1977) of L. nubifer J.L. Barnard, 1971 from the Cape Basin off southern Africa and the distribution of L. xenopus K.H. Barnard, 1931 in Antarctic waters is extended. A key to all species of Lepidepecreoides is presented. In Lepidepecreum five new species are described from Australian waters and the distributions of three species, L. foraminiferum Stebbing, 1888, L. infissum Andres, 1983 and $L$. urometacarinatum Andres, 1983, are extended within Antarctic waters.
\end{abstract}

LOWRY, J.K., \& H.E. STODDART, 2002. The lysianassoid amphipod genera Lepidepecreoides and Lepidepecreum in southern waters (Crustacea: Lysianassidae: Tryphosinae). Records of the Australian Museum 54(3): 335-364.

Only two species are currently known in the tryphosine genus Lepidepecreoides: L. nubifer J.L. Barnard, 1971, from the northeastern Pacific Ocean off the coast of Oregon and L. xenopus K.H. Barnard, 1931, from the Southern Ocean near South Georgia and the western side of the Antarctic Peninsula. Griffiths (1977) reported L. nubifer from the Cape Basin off southern Africa, but examination of his illustrations indicates a new species. Examination of collections made by the Lund University Chile Expedition (LUCE) (see Brattström \& Dahl, 1951) revealed a new species from southern Chile and examination of recent collections made in Australian waters by the Australian Museum and Museum Victoria indicated another two species living on the continental slope and bathyal plains off eastern Australia. Finally, examination of recent collections made in the Southern Ocean showed that the distribution of L. xenopus is more extensive than previously reported. All of this material is reported on here and a new key to the species of the genus is presented.

Barnard \& Karaman (1991) reported 28 species in the tryphosine genus Lepidepecreum. But within their list $L$. annulatum (Bate, 1862) and L. filiger (Stimpson, 1864) are unidentifiable species; L. nudum Imbach, 1967 has been transferred to Waldeckia by Lowry, 2000; and L. cingulatum K.H. Barnard, 1932 is probably a species of Orchomenella. Lowry \& Stoddart (2002) have re-diagnosed Lepidepecreum, described four new species (two from the eastern Andaman Sea and two from Japanese waters) and transferred L. magdalenensis (Shoemaker, 1942) and L. lukini (Budnikova, 1999) from Orchomenella to Lepidepecreum. One species, Lepidepecreum californiensis Vinogradov, 1994, was omitted from the list of species given by Lowry \& Stoddart (2002). 
Lepidepecreum has not previously been reported from Australian waters. Examination of collections made in southeastern and northwestern Australia by Museum Victoria revealed five new species. Examination of recent collections from the Southern Ocean showed more extensive distributions for L. foraminiferum Stebbing, 1888, L. infissum Andres, 1983 and L. urometacarinatum Andres, 1983, than were previously known. The description of the five new species here brings the total number of species in Lepidepecreum to 37.

Species of Lepidepecreum are easily recognizable because of their diamond-shaped bodies. Although species have been reported many times from most parts of the world virtually nothing is known about their behaviour. Lepidepecreum is related to genera such as Orchomenella, for which many species are known as demersal scavengers, but species of Lepidepecreum have never been reported from traps and are not considered to be scavengers. They have never been reported in association with other invertebrates.

Of the five species of Lepidepecreum known from Australian waters, four come from a small area of the continental slope off southeastern Australia. Although there is only a small amount of material known, these four species show a clear partitioning by depth: $L$. flindersi has been recorded from 120-400 m, L. freycineti from 600-800 m, L. tourville from 996-1850 $\mathrm{m}$ and L. baudini from 1840-2500 m depth.

In this paper we use the terminology introduced by Poore \& Lowry (1997) to describe the angle of the gnathopodal palms: "a palm that is close to perpendicular to the longitudinal axis of the propodus is referred to as transverse; a palm in which the angle is less than $90^{\circ}$ is referred to as acute; and a palm of more than $90^{\circ}$ is referred to as obtuse".

Material used in this study is lodged in the Australian Museum, Sydney (AM); Museum Victoria, Melbourne, Australia (NMV); the South African Museum, Cape Town (SAM); the Swedish Museum of Natural History, Stockholm (SMNH); and the United States Museum of Natural History, Washington, D.C. (USNM). The following abbreviations are used on the plates: A, antenna; E, epistome and upper lip; EP, epimeron; G, gnathopod; $\mathrm{H}$, head; MD, mandible; MP, maxilliped; MX, maxilla; $\mathrm{P}$, pereopod; T, telson; U, uropod; UR, urosomite; 1, left; r, right.

The descriptions are generated from a DELTA (Dallwitz et al., 1993, 1998) database of world tryphosine and uristid species. They are a compilation of all character variations which we recognise in these groups. Characters in italic text refer to the unique combination of characters which will diagnose each species against all others.

Implicit characters. To shorten the descriptions, and avoid repetition of commonly occurring character states, the following states are implicit throughout these descriptions, unless indicated otherwise or where the characters concerned are inapplicable.

Body not expanded to form a lateral bulge; without dorsal carina. Antenna 1 accessory flagellum not forming cap, terminal article not offset; calceoli small. Antenna 2 peduncular article 3 short; articles 3 to 5 not enlarged. Maxilliped basis without recurved hook. Pereonite 1 dorsally smooth. Gnathopod 1 without setal patch on merus and propodus; propodus small, sparsely setose along posterior margin, palm entire, straight. Pereonites 1-7 dorsally smooth. Pereopod 4 coxa without distinct lateral ridge. Pereopod 5 coxa without umbo; basis without photophore, not posteroproximally excavate, posterior margin not serrate, without mid-central spine. Pleonite 3 posterodorsal margin not produced. Urosomite 1 not projecting over urosomite 2 . Uropods 1 and 2 rami without stout striated robust setae. Uropod 2 inner ramus without constriction. Uropod 3 peduncle without dorsolateral flange; inner and outer rami well developed.

\section{Lepidepecreoides K.H. Barnard}

Lepidepecreoides K.H. Barnard, 1931: 426.-K.H. Barnard, 1932: 62.-J.L. Barnard, 1969: 347.-J.L. Barnard, 1971: 40.-Barnard \& Karaman, 1991: 495.

Diagnosis. Antenna 1 accessory flagellum 2-articulate, terminal article small, offset. Antenna 2 peduncular article 3 short. Gnathopod 1 carpus long (length 2 to $3.9 \times$ breadth). Pereopod 4 coxa with a weak posteroventral lobe (posterior margin concave). Pereopod 5 basis, posterior margin with mid-central spine.

Remarks. Originally K.H. Barnard (1931) considered the size of coxa 5 and the placement of the mandibular palp to be important defining characters for the genus. We have not found these characters to be informative. A character not previously considered is the length of peduncular article 3 on antenna 2. The elongate form of peduncular article 3 is a strong synapomorphy for the genus Lepidepecreum. Species of Lepidepecreoides have a short article 3, distinguishing them from species of Lepidepecreum. The elongate carpus of gnathopod 1 also appears to be a significant synapomorphy for some genera within the tryphosine group, a character Lepidepecreoides shares with Lepidepecreum, Tryphosella and Tryphosoides. Two apparent synapomorphies shared by Lepidepecreoides and Tryphosoides are the peculiar offset terminal article on the accessory flagellum and the loss of a well-developed posteroventral lobe on coxa 4. Only species of Lepidepecreoides have a mid-central spine on the basis of pereopod 5 . 


\section{Key to species of Lepidepecreoides}

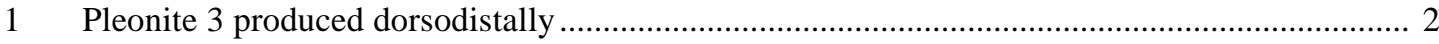

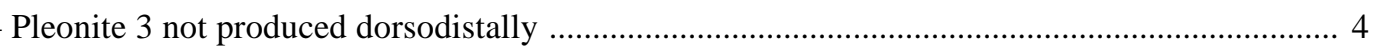

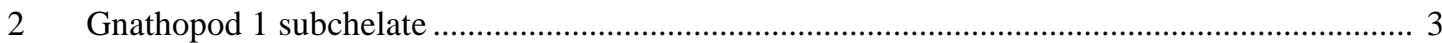

— Gnathopod 1 simple ................................................................................................... L. chincui

3 Pereopod 5 basis with rounded posteroventral lobe. Telson with apical robust setae

L. xenopus

Pereopod 5 basis with wedge-shaped posteroventral lobe. Telson

without apical robust setae

L. bassi

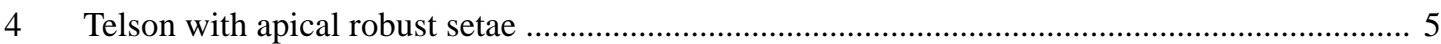

Telson without apical robust setae …………………..................................................... L. talboti

$5 \quad$ Gnathopod 1 palm acute. Coxa 5 without lateral ridge. Urosomite 1 produced dorsally into a recurved spine

L. nubifer

_ Gnathopod 1 palm slightly acute. Coxa 5 with distinct lateral ridge.

Urosomite 1 produced dorsally into a broad upright triangle

L. torresi

\section{Lepidepecreoides bassi n.sp.}

Figs. 1-3

Type material. HoLOTYPE, sex not known, $10.0 \mathrm{~mm}$, NMV J47713; 1 PARATYPE, sex not known, AM P57732; 76 km S of Point Hicks, Victoria, Australia, $38^{\circ} 29.33^{\prime} \mathrm{S} 149^{\circ} 19.98^{\prime} \mathrm{E}$, $1840 \mathrm{~m}$, sandy mud, fine shell, WHOI epibenthic sled, G.C.B. Poore et al., 26 October 1988, RV Franklin, stn SLOPE 69.

Type locality. $76 \mathrm{~km} \mathrm{~S}$ of Point Hicks, Victoria, Australia, $38^{\circ} 29.33^{\prime} \mathrm{S} 149^{\circ} 19.98^{\prime} \mathrm{E}, 1840 \mathrm{~m}$ depth.

Description. Based on holotype, sex not known, $10.0 \mathrm{~mm}$. Head with lateral cephalic lobe subtriangular, apically subacute; eyes apparently absent. Antenna 1 peduncular articles 1 and 2 without anterodistal lobe; accessory flagellum 2-articulate, with small terminal article offset; flagellum with strong 2-field callynophore; robust setae present on proximal articles; calceoli absent. Antenna 2 flagellum short; calceoli absent. Epistome/upper lip with epistome less produced than upper lip, straight; upper lip slightly produced, straight. Mandible molar columnar with oval, fully triturating surface; palp attached distally, article 3 with proximal A3-seta. Maxilla 1 outer plate with left and right setal-tooth 7 symmetrical, cuspidate distally along inner margin; palp distal margin with apical robust setae. Maxilliped outer plate with 2 (one long and slender, one short and broad) apical robust setae.

Gnathopod 1 subchelate; coxa large, about as long as coxa 2, subrectangular with straight anterior margin; basis without setae along anterior margin; ischium short; carpus

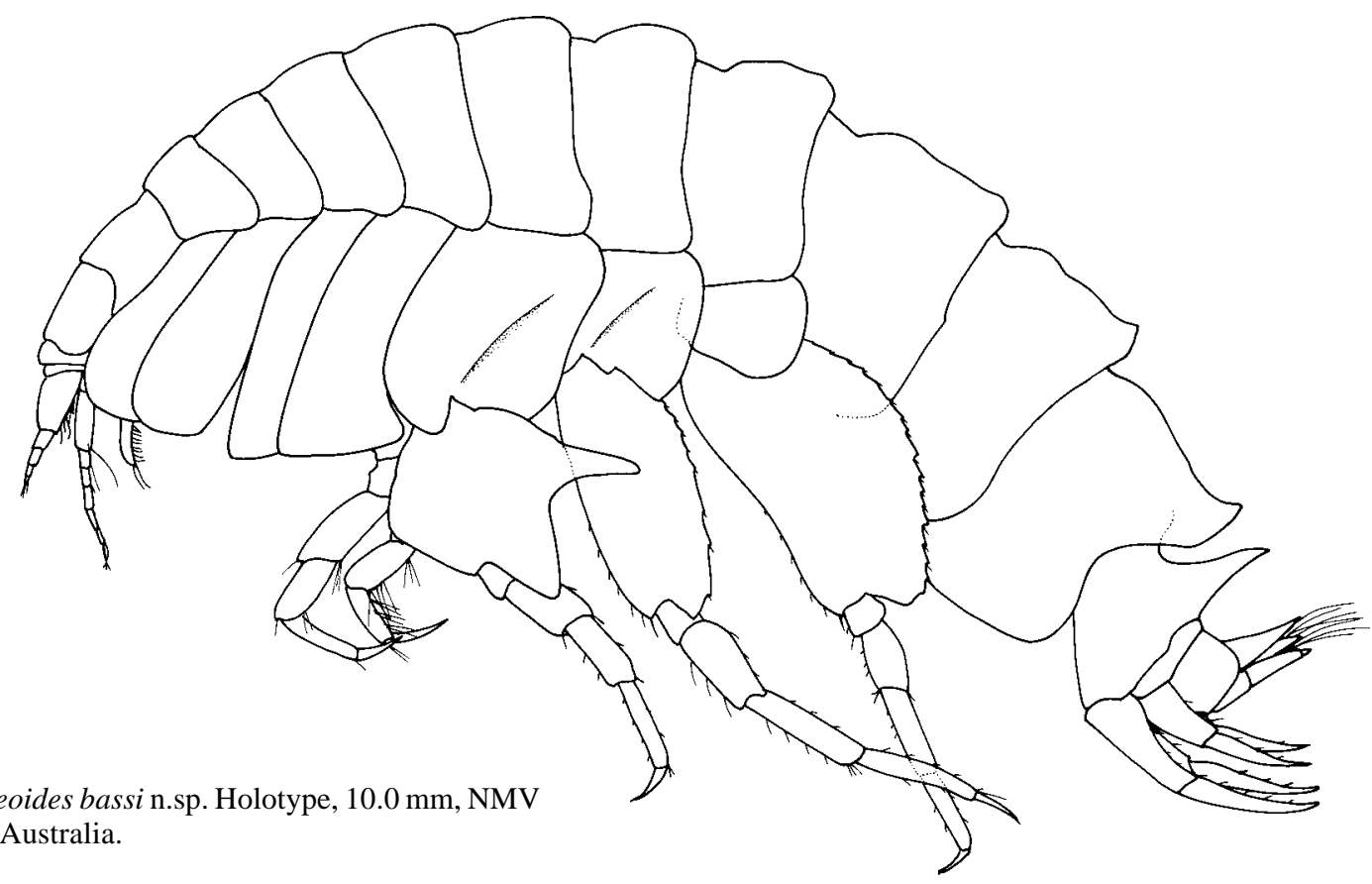

Figure 1. Lepidepecreoides bassi n.sp. Holotype, 10.0 mm, NMV J47713, Point Hicks, Australia. 


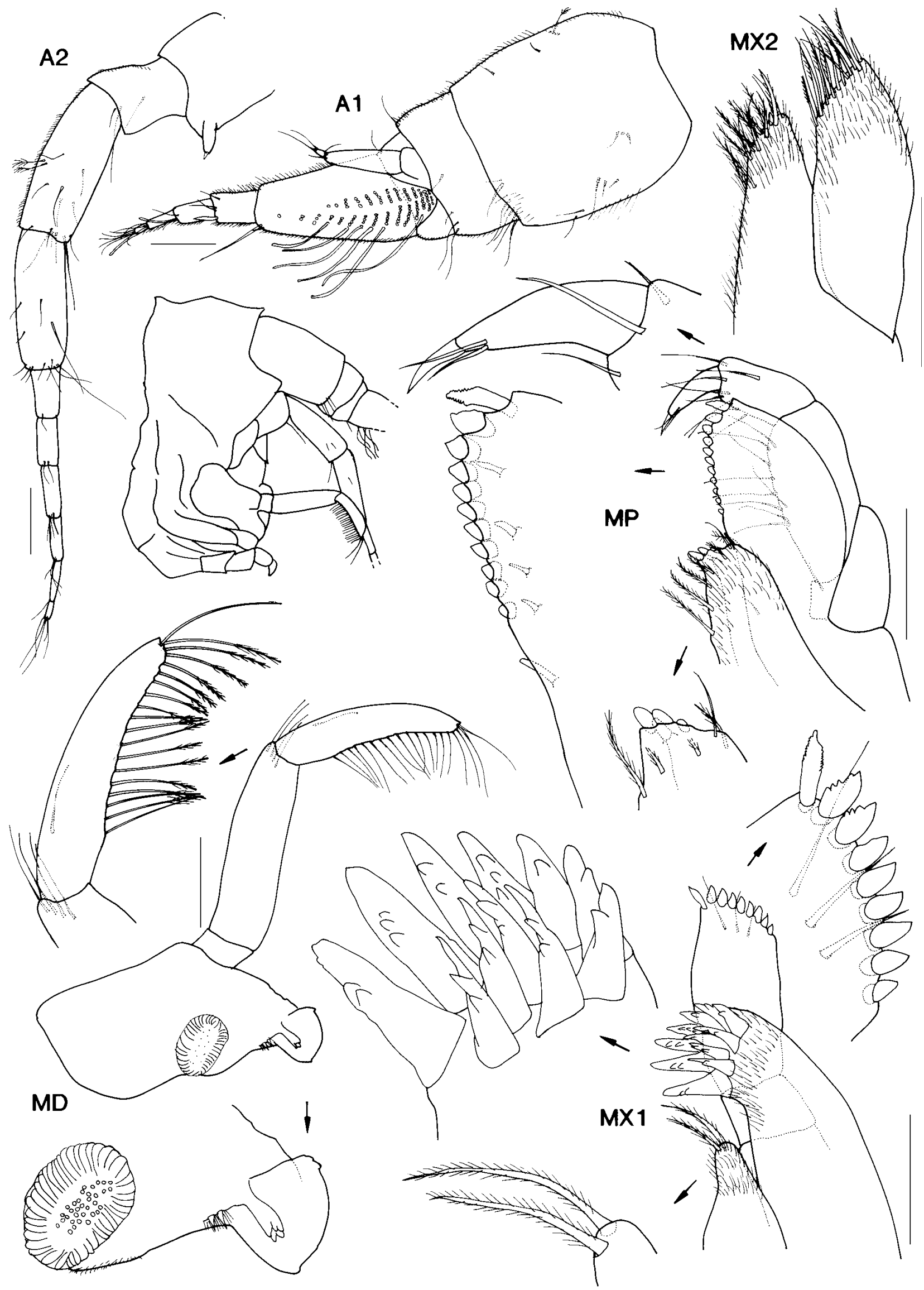

Figure 2. Lepidepecreoides bassi n.sp. Holotype, 10.0 mm, NMV J47713, Point Hicks, Australia. Scales represent 0.2 mm. 


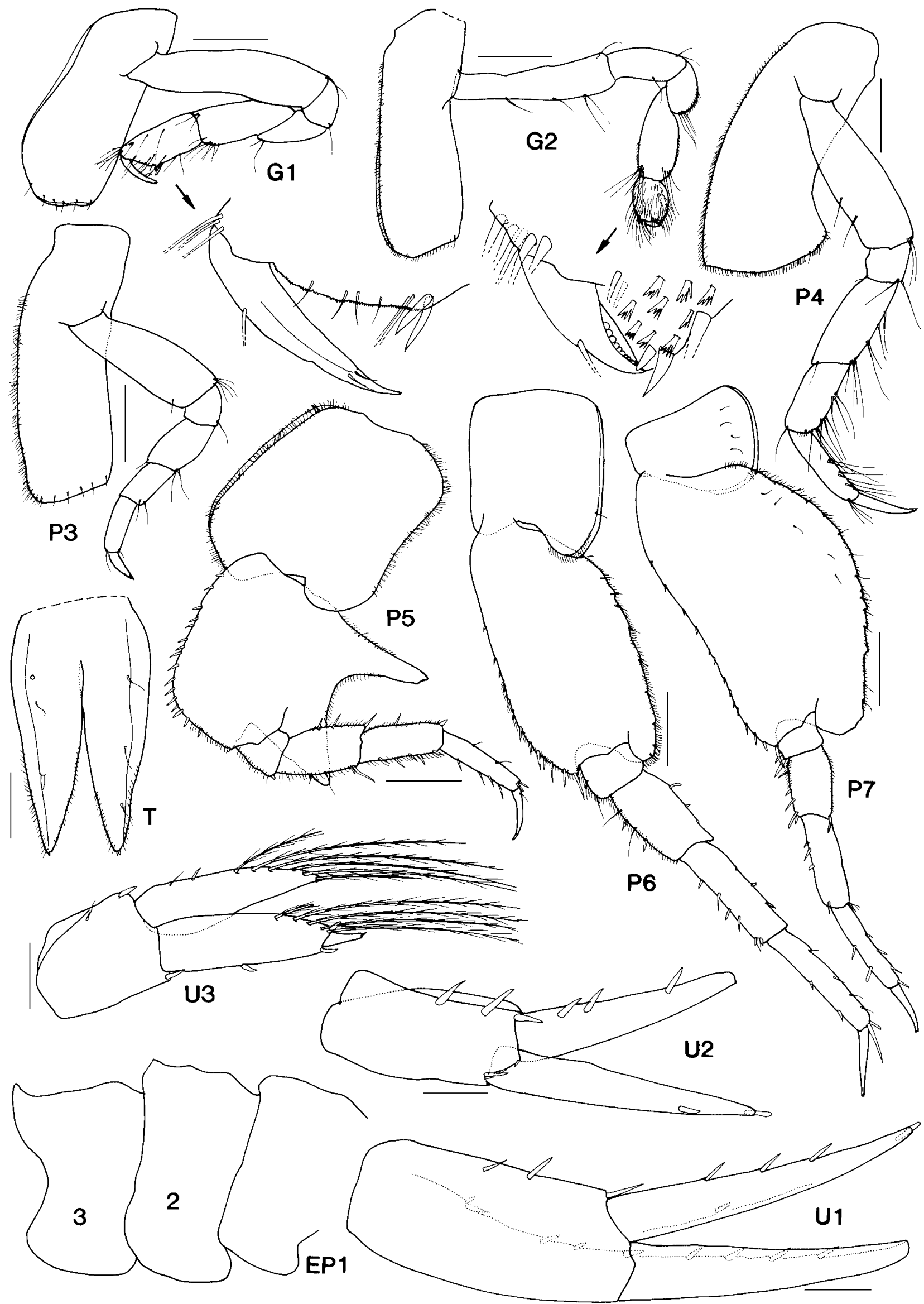

Figure 3. Lepidepecreoides bassi n.sp. Holotype, 10.0 mm, NMV J47713, Point Hicks, Australia. Scales for U13 , T represent $0.2 \mathrm{~mm}$, remainder represent $0.5 \mathrm{~mm}$. 
long $(2.6 \times$ breadth), longer than propodus, without posterior lobe; propodus small, margins subparallel, palm acute. Pereonite 2 with mid-dorsal carina. Gnathopod 2 palm slightly obtuse. Pereonite 3 with mid-dorsal carina. Pereonite 4 with mid-dorsal carina. Pereopod 4 coxa with a weak posteroventral lobe (posterior margin concave). Pereonite 5 with mid-dorsal carina. Pereopod 5 coxa with distinct lateral ridge; basis about as long as broad, posterior margin with mid-central spine, with well-developed wedgeshaped posteroventral lobe. Pereonite 6 with mid-dorsal carina. Pereonite 7 with mid-dorsal carina. Pereopod 7 basis posterodistally produced, not reaching merus, not posterodistally excavate.

Pleonite 1 with mid-dorsal carina. Pleonite 2 with middorsal carina. Pleonite 3 with mid-dorsal carina, dorsodistally produced, apically acute, posterodorsal margin produced. Epimeron 3 posterior margin smooth, posteroventral corner broadly rounded. Urosomite 1 with deep notch and a narrow vertically produced acute spine. Uropod 3 outer ramus article 2 short; with plumose setae on each ramus. Telson deeply cleft, with dorsal robust setae, without apical robust setae.

Etymology. Named after George Bass who, in December 1798 with Matthew Flinders in the sloop Norfolk, circumnavigated Van Diemans Land, so proving the existence of the strait, which now bears his name, between mainland Australia and Tasmania.

Remarks. Lepidepecreoides bassi is most similar to L. xenopus but is distinguished by the lack of anterodistal lobes on antenna 1 peduncle, the wedge-shaped posteroventral spine on pereopod 5 basis, the apically acute projection on pleonite 3 and the absence of apical robust setae on the telson.

Distribution. Bass Strait, Australia; 1840 m depth.

\section{Lepidepecreoides chincui n.sp.}

Figs. 4-6

Type material. HOLOTYPE, female, $5.8 \mathrm{~mm}$, non-ovigerous with long setose oostegites, SMNH 5250; 1 PARATYPE, juvenile, $3.5 \mathrm{~mm}$, SMNH 5251; Bahía Chincuí, Seno Reloneavi, southern Chile, eastern South Pacific Ocean, $41^{\circ} 32^{\prime} 00^{\prime \prime S} 73^{\circ} 01^{\prime} 30^{\prime \prime} \mathrm{W}, 70-80 \mathrm{~m}$, fine soft grey sand with small stones, 16 July 1949, LUCE stn M145A. -1 PARATYPE, 4.0 mm, AM P57733, Piedra Azul, NW of Punta Quillaipe, Seno Reloneavi, southern Chile, eastern South Pacific Ocean, 41 ${ }^{\circ} 31^{\prime} 30^{\prime \prime S} 72^{\circ} 48^{\prime} 15^{\prime \prime} \mathrm{W}, 30 \mathrm{~m}$, hard grey coarse sand, 14 December 1948, LUCE stn M16E.

Type locality. Bahía Chincuí, Seno Reloneavi, southern Chile,

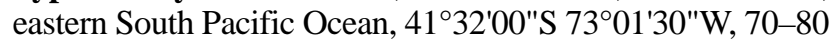
m depth.

Description. Based on holotype female, $5.8 \mathrm{~mm}$. Head with lateral cephalic lobe subtriangular, apically subacute; eyes apparently absent. Antenna 1 peduncular articles 1 and 2 without anterodistal lobe; accessory flagellum 2-articulate, with terminal article small, offset; flagellum with strong 2field callynophore; robust setae present on proximal articles; calceoli absent. Antenna 2 flagellum short, calceoli absent. Epistome/upper lip with epistome less produced than upper lip, straight; upper lip produced, rounded apically. Mandible molar columnar with oval, fully triturating surface; palp attached distally, article 3 with proximal A3-seta. Maxilla 1 outer plate with left and right setal-tooth 7 symmetrical, cuspidate distally along inner margin; palp distal margin with apical robust setae. Maxilliped outer plate with 2 (one long slender, one short broad) apical robust setae.

Gnathopod 1 simple; coxa large, about as long as coxa 2, subrectangular with straight anterior margin; basis sparsely setose along anterior margin; ischium short; carpus long $(3.0 \times$ breadth $)$, longer than propodus, without posterior

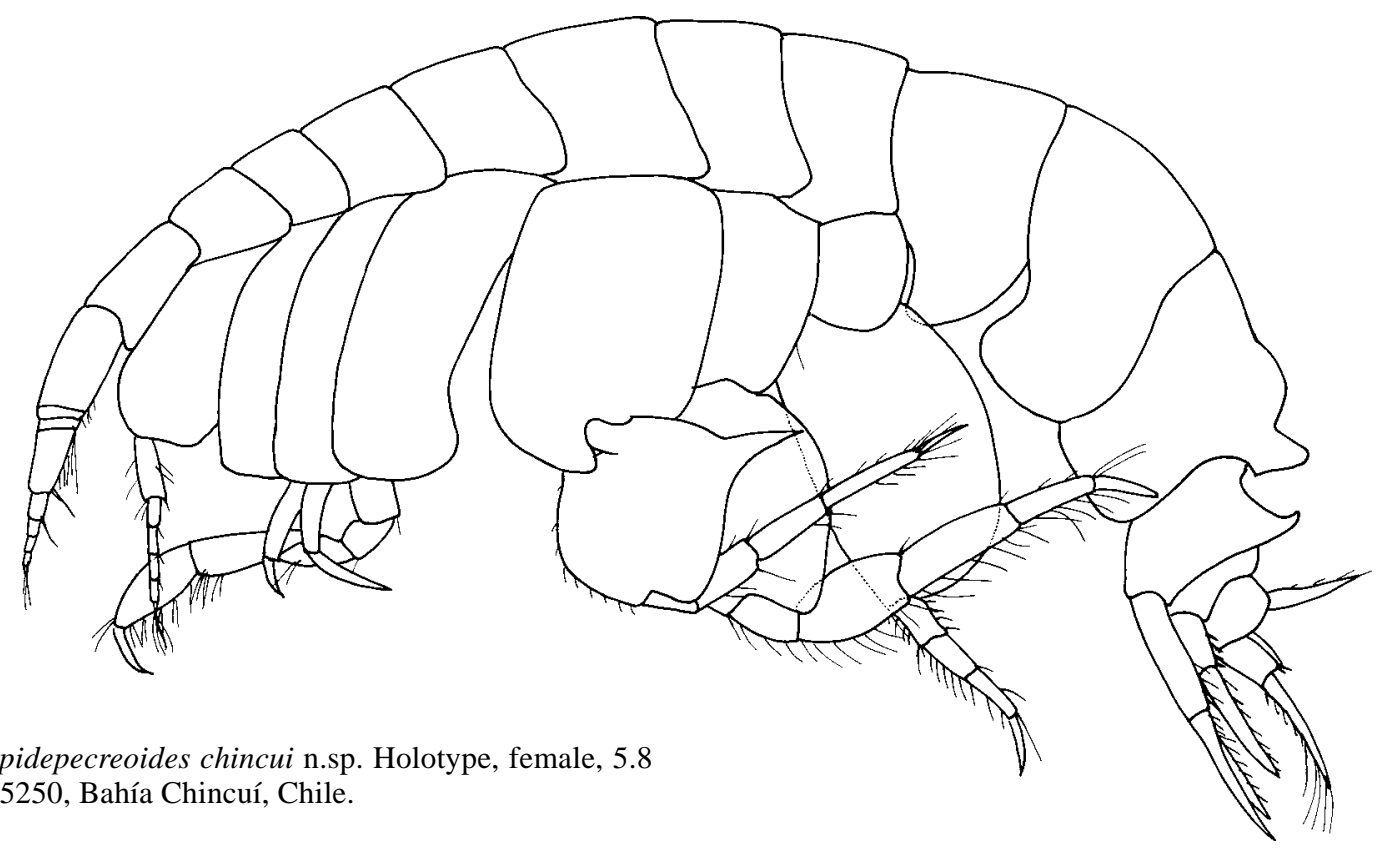

Figure 4. Lepidepecreoides chincui n.sp. Holotype, female, 5.8 mm, SMNH 5250, Bahía Chincuí, Chile. 


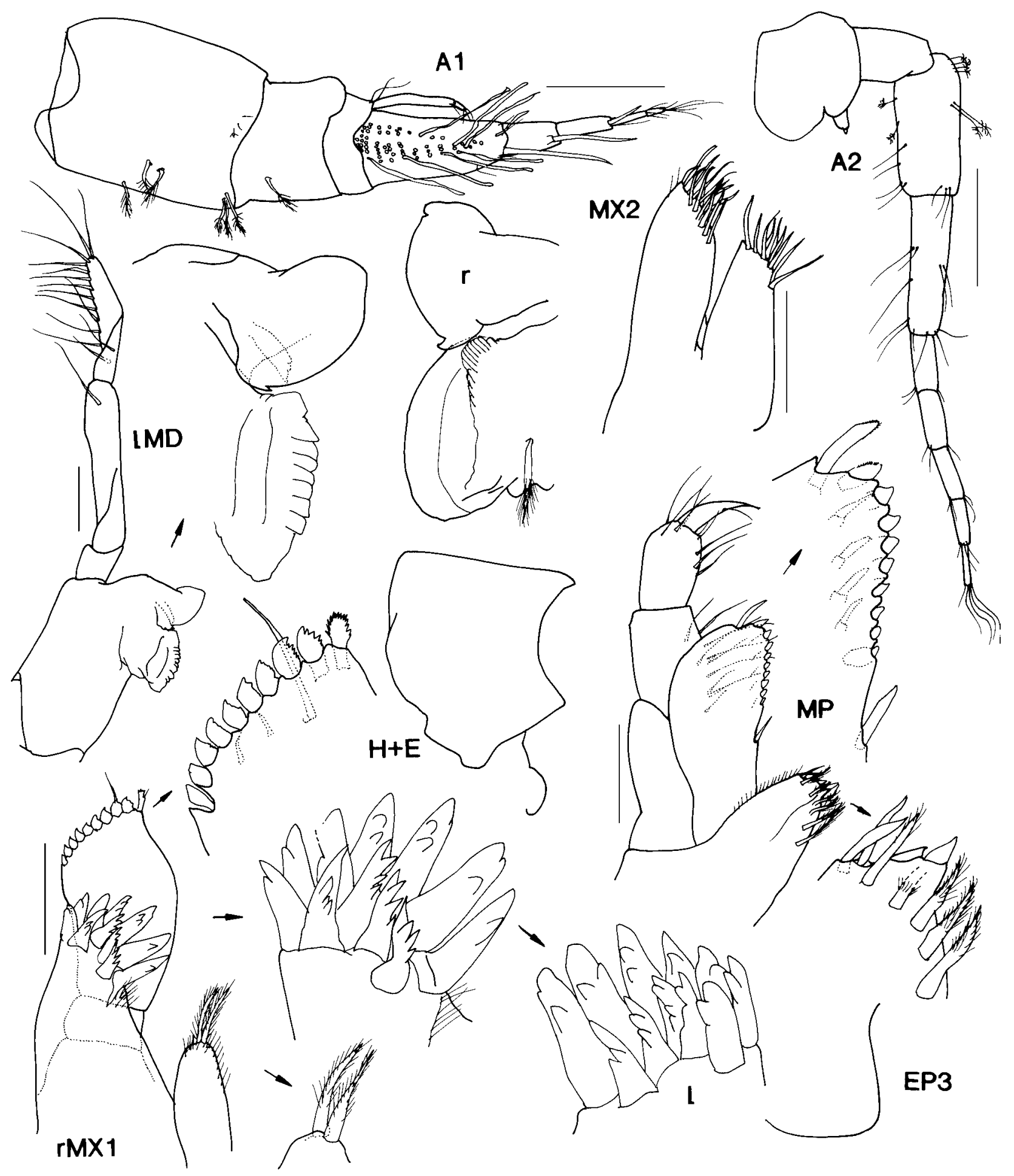

Figure 5. Lepidepecreoides chincui n.sp. Holotype, female, 5.8 mm, SMNH 5250, Bahía Chincuí, Chile. Scales represent 0.1 mm.

lobe; propodus small, margins subparallel, palm absent. Gnathopod 2 palm slightly obtuse. Pereonite 3 dorsally smooth. Pereonite 4 dorsally smooth. Pereopod 4 coxa with a weak posteroventral lobe (posterior margin concave). Pereonite 5 without mid-dorsal carina. Pereopod 5 coxa without distinct lateral ridge; basis about as long as broad, posterior margin with mid-central spine, with well-developed posteroventral lobe. Pereonite 6 dorsally smooth. Pereonite 7 dorsally smooth. Pereopod 7 basis posterodistally produced, not reaching merus, not posterodistally excavate.
Pleonite 1 dorsally smooth. Pleonite 2 dorsally smooth. Pleonite 3 with mid-dorsal carina, dorsally rounded, dorsodistally produced, apically rounded, posterodorsal margin produced. Epimeron 3 posterior margin smooth, posteroventral corner broadly rounded. Urosomite 1 without notch, with a narrow vertically produced recurved acute spine. Uropod 3 outer ramus article 2 short, with plumose setae on each ramus. Telson deeply cleft, with dorsal robust setae, with 1 long apical robust seta on each lobe. 


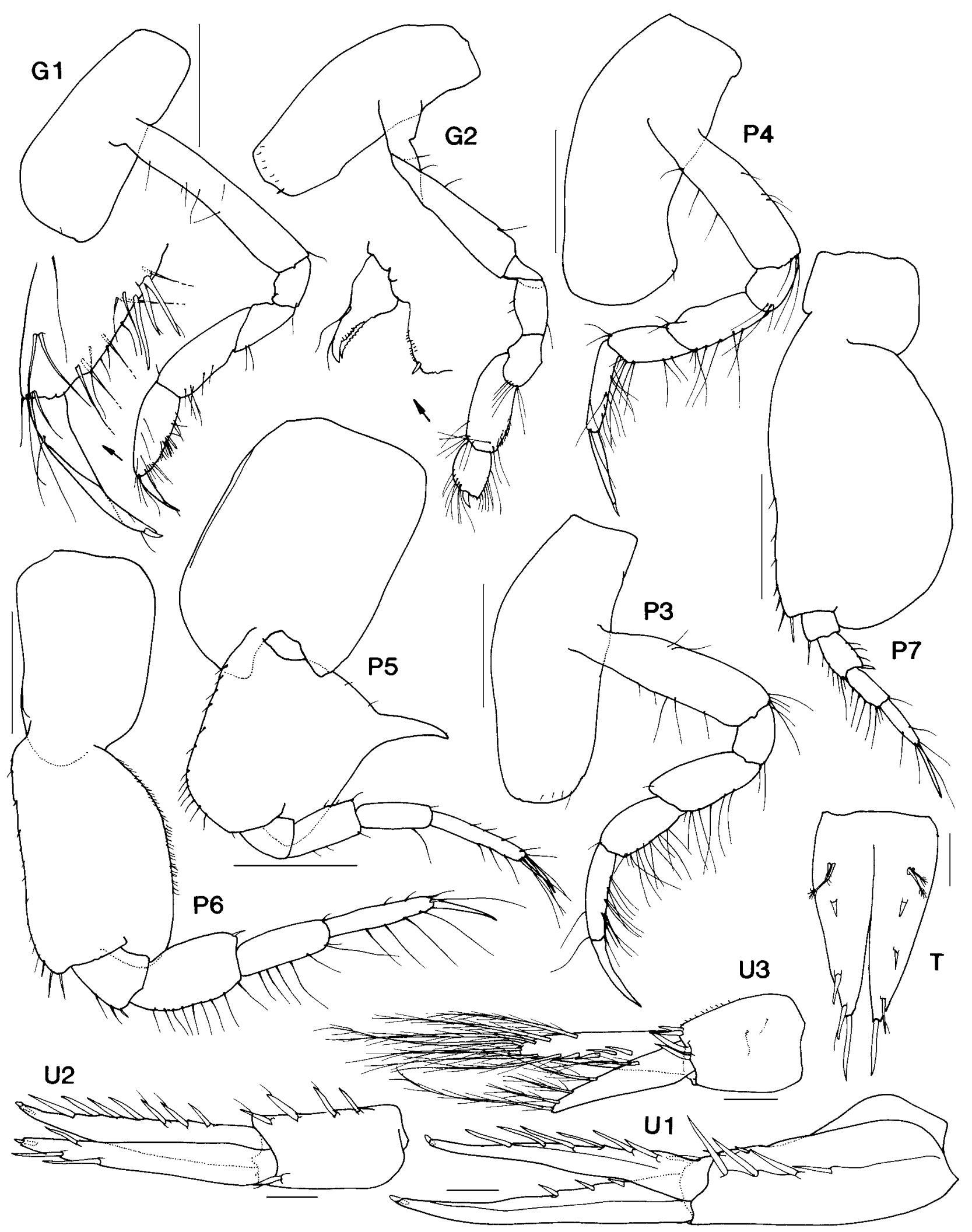

Figure 6. Lepidepecreoides chincui n.sp. Holotype, female, 5.8 mm, SMNH 5250, Bahía Chincuí, Chile. Scales for U1-3 represent $0.1 \mathrm{~mm}$, remainder represent $0.5 \mathrm{~mm}$. 
Etymology. Named after Bahía Chincuí, the type locality.

Remarks. Lepidepecreoides chincui is the only species of Lepidepecreoides with a simple gnathopod 1. It is also the only species recorded from less than $100 \mathrm{~m}$ depth.

Distribution. Southern Chile, eastern South Pacific Ocean; 30-80 m depth.

\section{Lepidepecreoides nubifer J.L. Barnard}

Lepidepecreoides nubifer J.L. Barnard, 1971: 41, figs. 26, 27.Austin, 1985: 600.-Barnard \& Karaman, 1991: 496. not Lepidepecreoides nubifer.-Griffiths, 1977: 98, fig. 4. (= L. talboti n.sp.).

Type locality. Off mouth of Columbia River, Oregon, USA, eastern North Pacific Ocean, $44^{\circ} 38.3^{\prime} \mathrm{N} 126^{\circ} 01.0^{\prime} \mathrm{W}, 2860 \mathrm{~m}$ depth.

Description. Head with lateral cephalic lobe subtriangular, apically subacute; eyes apparently absent. Antenna 1 peduncular articles 1 and 2 without anterodistal lobe; accessory flagellum 2-articulate, terminal article small, offset; flagellum with strong 2-field callynophore; calceoli absent. Antenna 2 flagellum short, calceoli absent. Epistome/upper lip with epistome less produced than upper lip, straight; upper lip produced, rounded apically. Mandible molar columnar with oval, fully triturating surface; palp attached distally, article 3 with proximal A3-seta. Maxilla 1 outer plate with setal-tooth 7 [symmetry not known], cuspidate distally along inner margin; palp distal margin with apical robust setae. Maxilliped outer plate with 1, long, slender apical robust setae.

Gnathopod 1 subchelate; coxa large, about as long as coxa 2, subrectangular with straight anterior margin; basis moderately setose along anterior margin; ischium short; carpus long $(2.5 \times$ breadth), longer than propodus, without posterior lobe; propodus small, margins subparallel, palm acute, entire, convex. Pereonite 2 dorsally smooth. Gnathopod 2 palm slightly obtuse. Pereonite 3 dorsally smooth. Pereonite 4 dorsally smooth. Pereopod 4 coxa with a weak posteroventral lobe (posterior margin concave). Pereonite 5 with mid-dorsal carina (slightly developed). Pereopod 5 coxa without distinct lateral ridge; basis about as long as broad, posterior margin with mid-central spine; with well-developed wedge-shaped posteroventral lobe. Pereonite 6 with mid-dorsal carina (slightly developed). Pereonite 7 with mid-dorsal carina (slightly developed). Pereopod 7 basis posterodistally produced, not reaching merus, posterodistally excavate.

Pleonite 1 with mid-dorsal carina. Pleonite 2 with middorsal carina. Pleonite 3 with mid-dorsal carina, not dorsodistally produced, posterodorsal margin produced. Epimeron 3 posterior margin smooth, posteroventral corner broadly rounded. Urosomite 1 with a narrow vertically produced recurved acute spine. Uropod 3 outer ramus article 2 short, with plumose setae on each ramus. Telson deeply cleft, with dorsal robust setae, with 1-2 apical robust setae on each lobe.

Distribution. Oregon, USA, eastern North Pacific Ocean; $2860 \mathrm{~m}$ depth.

\section{Lepidepecreoides talboti n.sp.}

Lepidepecreoides nubifer.-Griffiths, 1977: 98, fig. 4.

Type material. HOLOTYPE, female, 17 mm, SAM A10547, [west of Cape Point, South Africa, southeastern South Atlantic Ocean], $34^{\circ} 36^{\prime} \mathrm{S} 17^{\circ} 00^{\prime} \mathrm{E}, 2740 \mathrm{~m}$, F.H. Talbot, 10 December 1959, RS Africana II [material recorded by Griffiths, 1977 as Lepidepecreoides nubifer].

Type locality. West of Cape Point, South Africa, southeastern South Atlantic Ocean, $34^{\circ} 36^{\prime} \mathrm{S} 17^{\circ} 00^{\prime} \mathrm{E}, 2740$ $\mathrm{m}$ depth.

Description. Based on holotype female, $17 \mathrm{~mm}$. Body [shape not known]. Head with lateral cephalic lobe subtriangular, apically subacute; eyes apparently absent. Antenna 1 peduncular articles 1 and 2 without anterodistal lobe; accessory flagellum 2-articulate, terminal article small, offset; flagellum with strong 2-field callynophore; robust setae present on proximal articles; calceoli absent. Antenna 2 [not known]. Epistome/upper lip [not known]. Mandible molar columnar with oval, fully triturating surface; palp attached extremely distally, article 3 without proximal A3setae. Maxilla 1 outer plate with setal-tooth 7 [symmetry not known], cuspidate distally along inner margin; palp distal margin with apical robust setae. Maxilliped outer plate with 2 long, slender apical robust setae.

Gnathopod 1 subchelate; coxa large, about as long as coxa 2, subrectangular with straight anterior margin; basis sparsely setose along anterior margin; ischium short; carpus long $(3.1 \times$ breadth $)$, longer than propodus, without posterior lobe; propodus small, margins subparallel, palm acute, entire, straight. Pereonite 2 [not known]. Gnathopod 2 palm slightly obtuse. Pereonites 3-6 [dorsal margins unknown]. Pereopod 5 coxa without distinct lateral ridge; basis longer than broad, posterior margin with mid-central spine, with well-developed slender posteroventral lobe. Pereonite 7 with mid-dorsal carina (slightly developed). Pereopod 7 [unknown].

Pleonite 1 with mid-dorsal carina. Pleonite 2 with dorsal carina, rounded dorsodistally. Pleonite 3 with mid-dorsal carina, with irregular mid-dorsal margin, posterodorsal margin produced. Epimeron 3 [unknown]. Urosomite 1 with mid-dorsal, tube-shaped carina. Uropod 3 outer ramus article 2 short, with plumose setae on each ramus. Telson deeply cleft, without dorsal robust setae, without apical robust setae.

Etymology. Named for Dr Frank Talbot, collector of the type material, renowned zoologist and yachtsman, and previous Director of both the South African Museum and the Australian Museum.

Remarks. Lepidepecreoides talboti was considered by Griffiths (1977) to be the same species as L. nubifer J.L. Barnard, 1971. Griffiths considered the morphological differences between his material and that of Barnard to be not taxonomically significant. Based on our examination of known species in the genus and the geographical distance between the two populations, we consider the taxa to be separate species. Lepidepecreoides talboti differs from $L$. nubifer in the length of the gnathopod 1 carpus, the dorsal shape of the pleonites and the setation of the telson.

We have assumed that the segments of the dorsal profile in figure 4 I of Griffiths (1977) have been mis-labelled, and 
that the segment labelled " 3 " is really urosomite 1 , etc. It would otherwise seem inconceivable that Griffiths would identify his material as L. nubifer, which has no strong dorsal projection on pleonite 3 .

Distribution. Cape Basin, southeastern South Atlantic Ocean; $2740 \mathrm{~m}$ depth.

\section{Lepidepecreoides torresi n.sp.}

Figs. 7-9

Type material. HolOTYPe, female, with non-setose oostegites, $6.8 \mathrm{~mm}$, AM P57734, E of Cape York, Australia, Coral Sea, $10^{\circ} 32.72 ' \mathrm{~S} 144^{\circ} 12.8^{\prime} \mathrm{E}, 780-795 \mathrm{~m}$, epibenthic sled, P. Hutchings et al., 20 August 1988, RV Franklin, stn 06/88-03.

Type locality. East of Cape York, Australia, Coral Sea, $10^{\circ} 32.72 ' \mathrm{~S} 144^{\circ} 12.8^{\prime} \mathrm{E}, 780-795 \mathrm{~m}$ depth.

Description. Based on holotype female, $6.8 \mathrm{~mm}$. Head with lateral cephalic lobe subtriangular, apically subacute; eyes apparently absent. Antenna 1 peduncular articles 1 and 2 without anterodistal lobe; accessory flagellum 2-articulate, terminal article small, offset; flagellum with strong 2-field callynophore; robust setae present on proximal articles; calceoli absent. Antenna 2 flagellum short, calceoli absent. [Epistome/upper lip unknown]. Mandible molar columnar with oval, fully triturating surface; palp attached distally, article 3 with proximal A3-seta. Maxilla 1 outer plate with left and right setal-tooth 7 symmetrical, cuspidate distally along inner margin; palp distal margin with apical robust setae. Maxilliped outer plate with 2 (one slender, one broad) apical robust setae.

Gnathopod 1 subchelate; coxa large, about as long as coxa 2, subrectangular with straight anterior margin; basis sparsely setose along anterior margin; ischium short; carpus long $(2.3 \times$ breadth $)$, longer than propodus, without posterior lobe; propodus small, margins subparallel, palm slightly acute. Pereonite 2 without mid-dorsal carina. Gnathopod 2 palm transverse. Pereonite 3 without mid-dorsal carina. Pereonite 4 without mid-dorsal carina. Pereopod 4 coxa with a weak posteroventral lobe (posterior margin concave). Pereonite 5 without mid-dorsal carina. Pereopod 5 coxa with distinct lateral ridge; basis about as long as broad, posterior margin with mid-central spine, with welldeveloped wedge-shaped posteroventral lobe. Pereonite 6 without mid-dorsal carina. Pereonite 7 without mid-dorsal carina. Pereopod 7 basis posterodistally produced, not reaching merus, not posterodistally excavate.

Pleonite 1 without mid-dorsal carina. Pleonite 2 without mid-dorsal carina. Pleonite 3 with mid-dorsal carina, not dorsodistally produced, posterodorsal margin produced. Epimeron 3 posterior margin smooth, posteroventral corner broadly rounded. Urosomite 1 with deep notch and subtriangular, acutely produced boss. Uropod 3 outer ramus article 2 short; with plumose setae on each ramus. Telson deeply cleft, with dorsal robust setae, with 1 apical robust seta on each lobe.

Etymology. Named for Luis Vaez de Torres, commander of the San Pedro, which, in 1606, was the first European vessel to sail through the strait that now bears Torres' name.

Distribution. Coral Sea, western South Pacific Ocean; 780$795 \mathrm{~m}$ depth.

\section{Lepidepecreoides xenopus K.H. Barnard}

Fig. 10

Lepidepecreoides xenopus K.H. Barnard, 1931: 426.-K.H. Barnard, 1932: 62, fig. 24.-J.L. Barnard, 1958: 93.-Thurston \& Allen, 1969: 360.-Lowry \& Bullock, 1976: 93.-Barnard \& Karaman, 1991: 496.-Ren \& Huang, 1991: 247, fig. 37.-De Broyer \& Jazdzewski, 1993: 15, 69.

Material examined. Three specimens, 4.0-4.5 mm, and 1 partial specimen, USNM 306582, Weddell Sea, Antarctica, $74^{\circ} 07^{\prime} \mathrm{S} 39^{\circ} 38^{\prime} \mathrm{W}, 731 \mathrm{~m}$, epibenthic sled, 6 February 1968 , USCGC Glacier, IWSOE cruise 1, stn 0001. — 1 male, 9.5 mm, NMV J47714, off Enderby Land, Antarctica, Southern Ocean, $65^{\circ} 50.10^{\prime} \mathrm{S} 50^{\circ} 34.30^{\prime} \mathrm{E}$ to $65^{\circ} 50.30^{\prime} \mathrm{S} 50^{\circ} 34.90^{\prime} \mathrm{E}$, $540 \mathrm{~m}$, rock with mud and clay patches, WHOI epibenthic sled, M. Norman, MS Nella Dan, stn HRD 011. _ 1 specimen, $6.0 \mathrm{~mm}$, NMV J38225, Amery Depression, Antarctica, $68^{\circ} 04.36$ 'S $72^{\circ} 17.81^{\prime} \mathrm{E}$ to $68^{\circ} 04.44^{\prime} \mathrm{S} 72^{\circ} 18.11$ 'E, $765 \mathrm{~m}$, epibenthic sled, 17 February 1993, M. O'Loughlin on RSV Aurora Australis, stn AA93-151.

Figure 7. Lepidepecreoides torresi n.sp. Holotype, female, 6.8 mm, AM P57734, Cape York, Australia.

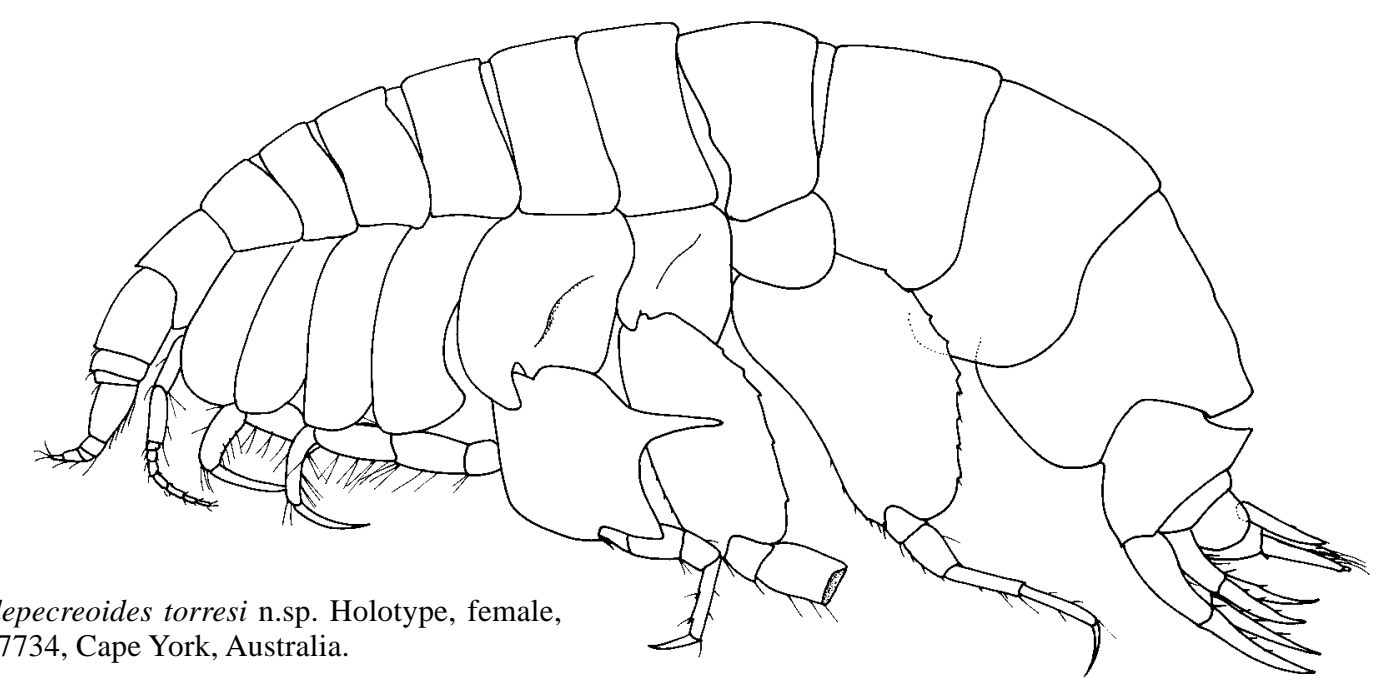




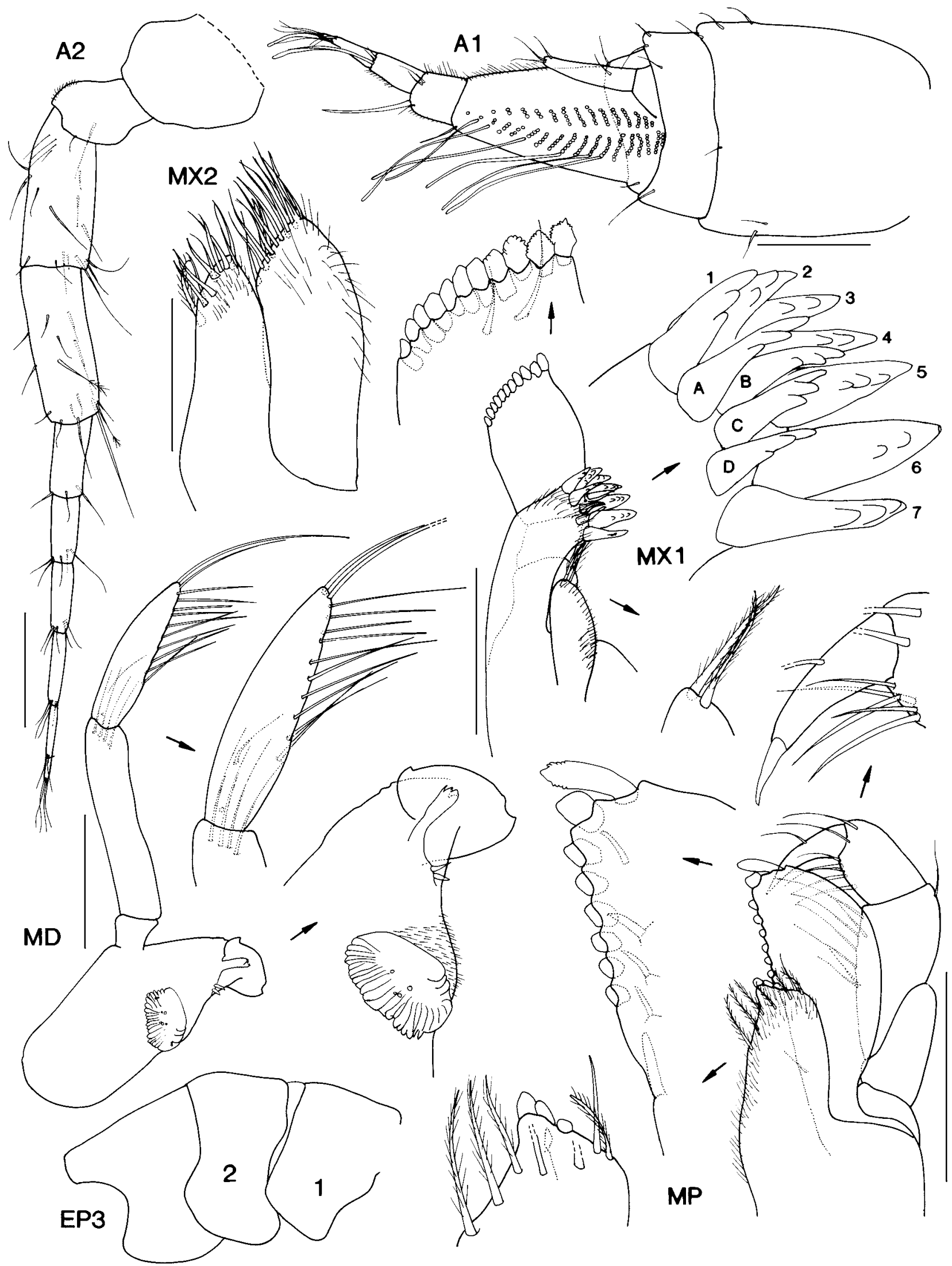

Figure 8. Lepidepecreoides torresi n.sp. Holotype, female, 6.8 mm, AM P57734, Cape York, Australia. Scales represent 0.2 mm.

Type locality. Schollaert Channel, Palmer Archipelago,

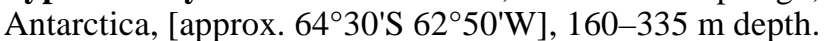

Description. Head with lateral cephalic lobe subtriangular, apically subacute; eyes apparently absent. Antenna 1 peduncular articles 1 and 2 with weakly developed anterodistally rounded lobe; accessory flagellum 2articulate, terminal article small, offset; flagellum with 


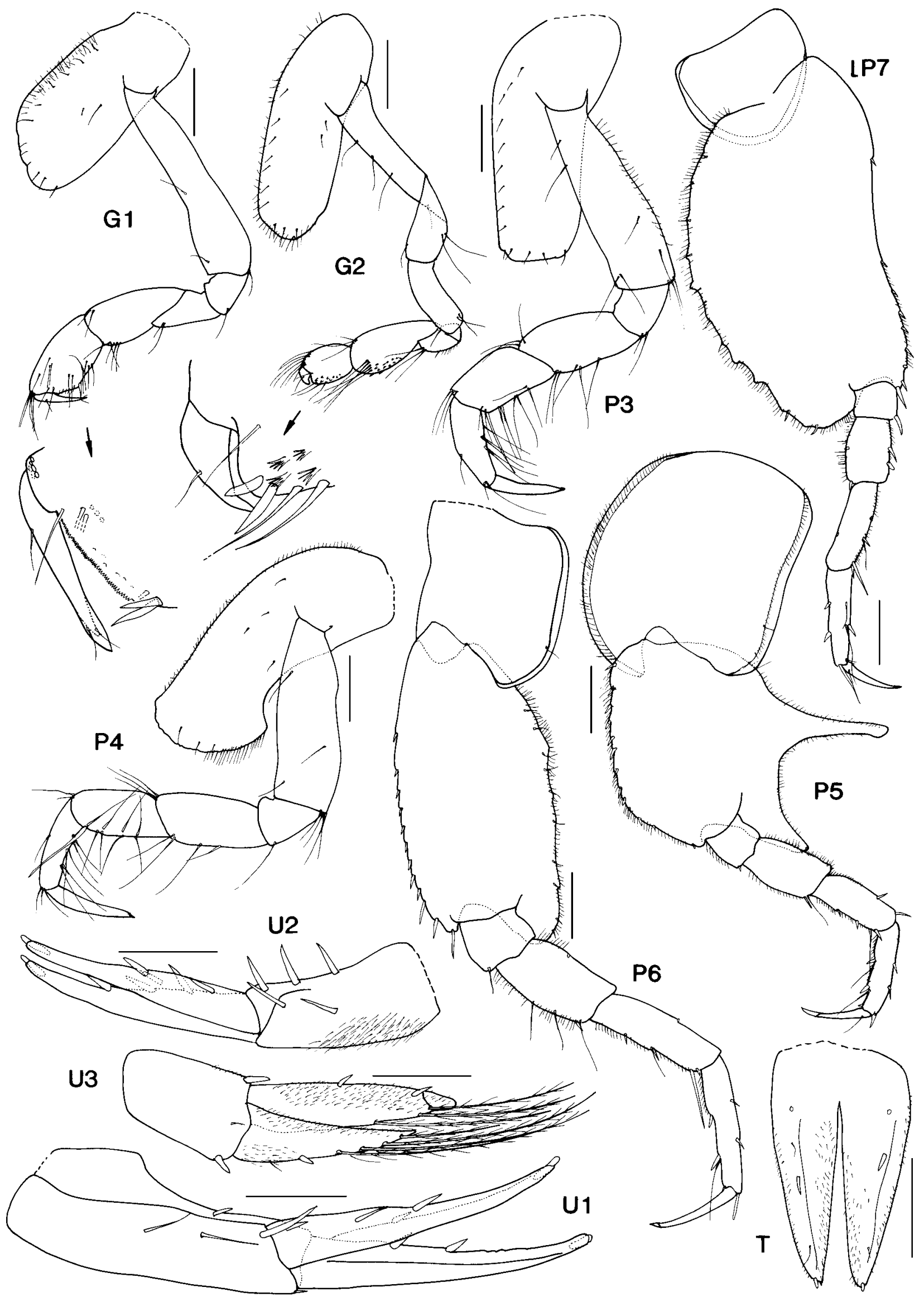

Figure 9. Lepidepecreoides torresi n.sp. Holotype, female, 6.8 mm, AM P57734, Cape York, Australia. Scales represent 0.2 mm. 


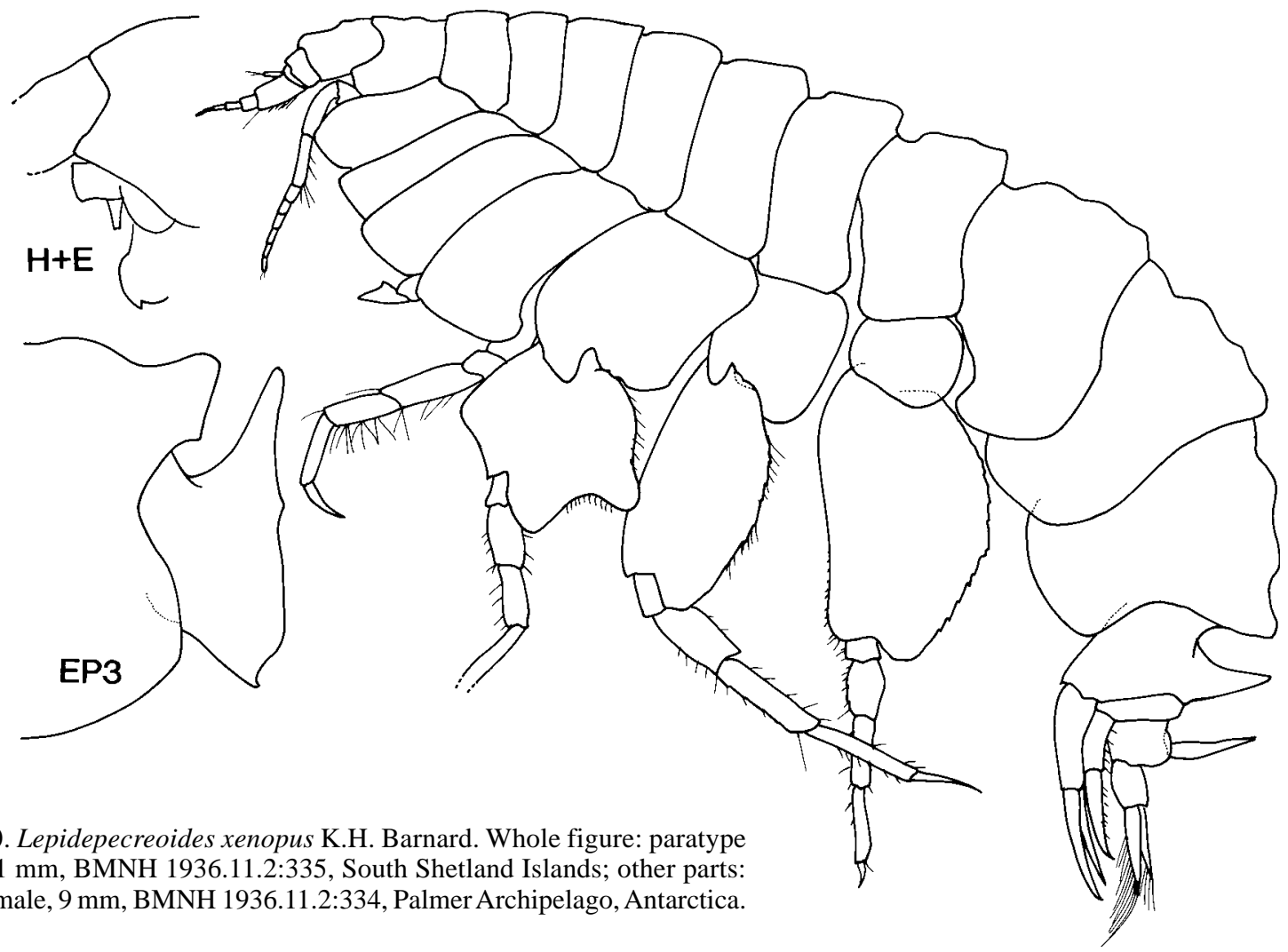

Figure 10. Lepidepecreoides xenopus K.H. Barnard. Whole figure: paratype female, $11 \mathrm{~mm}$, BMNH 1936.11.2:335, South Shetland Islands; other parts: holotype male, 9 mm, BMNH 1936.11.2:334, Palmer Archipelago, Antarctica.

strong 2-field callynophore; robust setae present on proximal articles; calceoli absent. Antenna 2 flagellum short, calceoli absent. Epistome/upper lip with upper lip slightly produced, straight. Mandible molar columnar, with subcircular or oval, fully triturating surface; palp attached distally, article 3 with proximal A3-seta. Maxilla 1 outer plate with setal-tooth 7 cuspidate distally along inner margin; palp distal margin with apical robust setae. Maxilliped outer plate with 2, long, (one slender, one broad) apical robust setae.

Gnathopod 1 subchelate; coxa large, about as long as coxa 2, subrectangular with straight anterior margin; basis sparsely setose along anterior margin; ischium short; carpus long $(2.4 \times$ breadth $)$, longer than propodus, without posterior lobe; propodus small, margins subparallel, palm acute. Gnathopod 2 palm slightly obtuse. Pereonite 3 with middorsal carina. Pereonite 4 with mid-dorsal carina. Pereopod 4 coxa with a weak posteroventral lobe (posterior margin concave). Pereonite 5 with mid-dorsal carina. Pereopod 5 coxa with distinct lateral ridge; basis about as long as broad, posterior margin with mid-central spine; with welldeveloped posteroventral lobe. Pereonite 6 with mid-dorsal carina. Pereonite 7 with mid-dorsal carina. Pereopod 7 basis posterodistally produced, not reaching merus, not posterodistally excavate.

Pleonite 1 with mid-dorsal carina. Pleonite 2 with middorsal carina. Pleonite 3 with mid-dorsal carina, dorsodistally produced, apically rounded, posterodorsal margin produced. Epimeron 3 posterior margin smooth, posteroventral corner broadly rounded. Urosomite 1 with a narrow vertically produced recurved acute spine. Uropod 3 outer ramus article 2 short, with plumose setae on each ramus. Telson deeply cleft, without dorsal robust setae, with 1 apical robust seta on each lobe.
Remarks. We have examined the type material of $L$. xenopus and, in all three specimens, the dorsal projection on pleonite 3 is more rounded than indicated in K.H. Barnard's whole-animal figure of the male holotype. All six specimens recorded here have similar projections on pleonite 3 and urosomite 1, and a rounded posteroventral lobe on pereopod 5 basis. Five of the six specimens have a well-developed spine on the mid-posterior margin of pereonite 5 basis, as K.H. Barnard showed for his "juvenile" (an immature male). The $6 \mathrm{~mm}$ specimen from the Amery Depression has a rounded lobe on the posterior margin, similar to that of the holotype male and paratype female.

Distribution. Antarctic Peninsula, South Georgia, South Shetland Islands, Weddell Sea, Enderby Land and Amery Depression, Antarctica; 130-1080 m depth.

\section{Lepidepecreum Bate \& Westwood}

Diagnosis. Body expanded to form a lateral bulge (not known for all species). Antenna 2 peduncular article 3 elongate. Mandible molar columnar (sometimes reduced) oval, fully triturating. Gnathopod 1 coxa large, about as long as coxa 2 (more than $0.7 \times$ ); carpus long (length 2 to $3.9 \times$ breadth). Uropod 3 outer ramus 2 -articulate.

\section{Lepidepecreum baudini n.sp.}

Figs. 11-13

Type material. HOLOTYPE, female, 5.5 mm, NMV J47715; 1 PARATYPE, male, $7.5 \mathrm{~mm}$, NMV J47716; 1 PARATYPE, immature male, 6.5 mm, NMV J47717; 14 PARATYPES, NMV J47718; 5 PARATYPES, AM P57735; $76 \mathrm{~km} \mathrm{~S}$ of Point Hicks, Victoria, 
Figure 11. Lepidepecreum baudini n.sp. Paratype immature male, 6.5 mm, NMV J47717, Point Hicks, Australia.

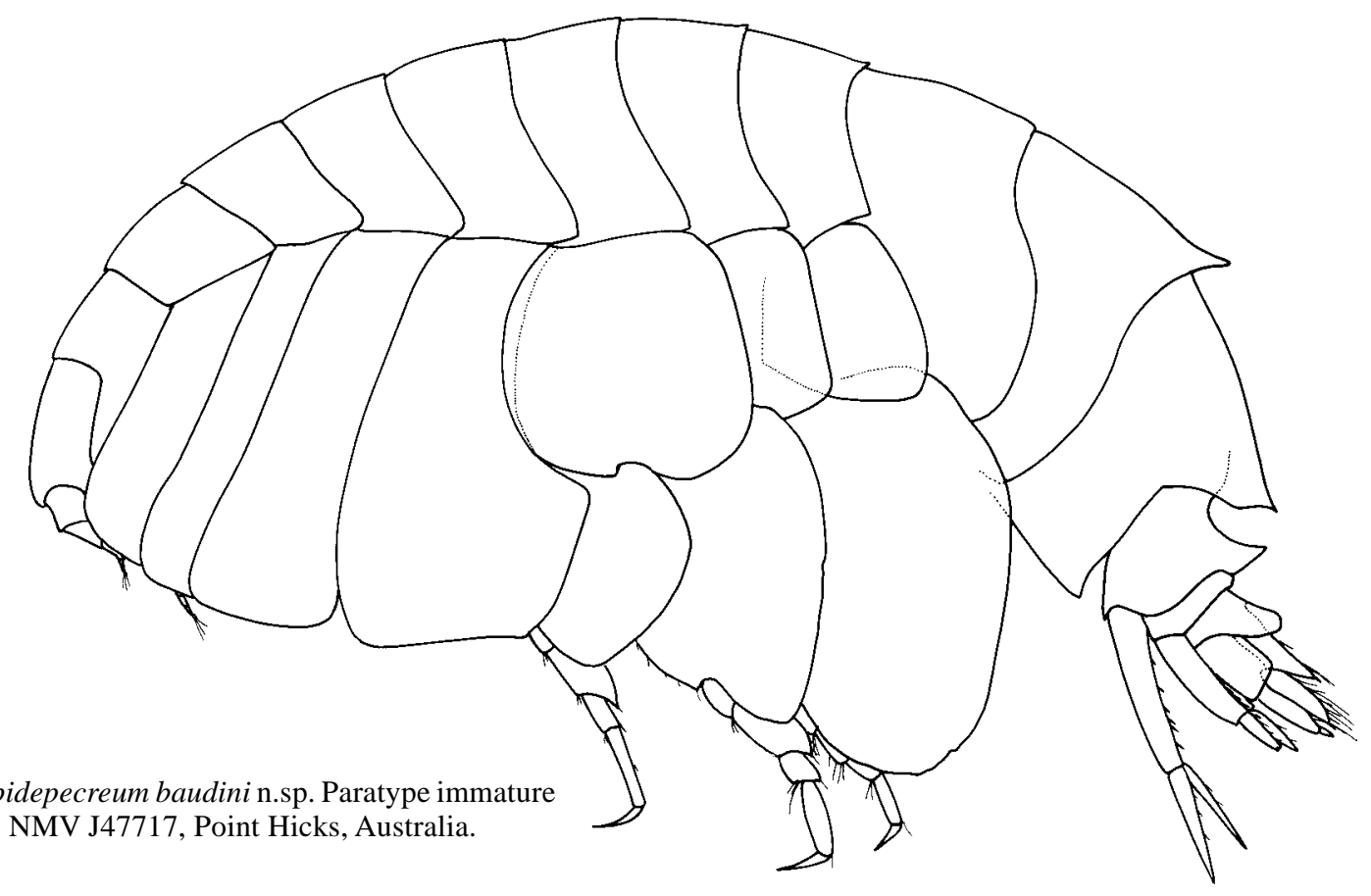

Australia, $38^{\circ} 29.33^{\prime} \mathrm{S} 149^{\circ} 19.98^{\prime} \mathrm{E}, 1840 \mathrm{~m}$, sandy mud, fine shell, WHOI epibenthic sled, G.C.B. Poore et al., 26 October 1988, RV Franklin stn SLOPE 69. - 1 PARATYPE, NMV J47719, 96 km S of Point Hicks, Victoria, Australia, 38 ${ }^{\circ} 40.29^{\prime} \mathrm{S}$ $149^{\circ} 18.06 ' \mathrm{E}, 2900 \mathrm{~m}$, compacted clay, WHOI epibenthic sled, G.C.B. Poore et al., 25 October 1988, RV Franklin stn SLOPE 66. - 1 PARATYPE, AM P57736, E of Cape Naturaliste, Tasmania, Australia, $40^{\circ} 45.94 ' \mathrm{~S} 149^{\circ} 01.62^{\prime} \mathrm{E}$ to $40^{\circ} 46.54$ 'S $149^{\circ} 00.27 ' E, 2400-2500$ m, mud, clay, R.T. Springthorpe, 10 December 1986, RV Franklin stn 1086-04.

Type locality. $76 \mathrm{~km} \mathrm{~S}$ of Point Hicks, Victoria, Australia, $38^{\circ} 29.33^{\prime} \mathrm{S} 149^{\circ} 19.98^{\prime} \mathrm{E}, 1840 \mathrm{~m}$ depth.

Description. Based on holotype female, $5.5 \mathrm{~mm}$. Body expanded to form a lateral bulge. Head with lateral cephalic lobe a semidome, apically subacute. Antenna 1 peduncular articles 1 and 2 without anterodistal lobe; accessory flagellum 2-articulate; flagellum without callynophore; robust setae absent from proximal articles; calceoli absent. Antenna 2 peduncular article 3 elongate; flagellum short, calceoli absent. Epistome/upper lip with epistome produced beyond upper lip, broadly rounded. Mandible molar columnar with oval, fully triturating surface; mandibular palp attached proximally, article 3 without proximal A3-setae. Maxilla 1 outer plate with setaltooth 7 present, left and right asymmetrical, cuspidate distally; palp distal margin with apical robust setae. Maxilliped outer plate with 1 short, slender apical robust seta.

Gnathopod 1 subchelate; coxa large, about as long as coxa 2, subrectangular with straight anterior margin; basis moderately setose along anterior margin; ischium short; carpus long, subequal in length to propodus, without posterior lobe; propodus with margins subparallel, palm acute, entire, concave. Gnathopod 2 palm slightly obtuse. Pereopod 4 coxa without distinct lateral ridge, with a welldeveloped posteroventral lobe. Pereonite 5 dorsally smooth.
Pereopod 5 coxa without distinct lateral ridge, without umbo, basis about as long as broad. Pereopod 7 basis posterodistally produced beyond merus.

Pleonite 1 dorsally smooth, not produced dorsodistally. Pleonite 2 with mid-dorsal carina, acutely produced posterodistally. Pleonite 3 with mid-dorsal carina, produced dorsodistally, apically acute, posterodorsal margin produced. Epimeron 3 posterior margin smooth, posteroventral corner acutely produced. Urosomite 1 with a narrow vertically produced recurved acute spine. Uropod 3 inner and outer rami well developed; outer ramus article 2 short; without plumose setae on rami. Telson moderately cleft, with dorsal robust setae, with 1 apical robust seta on each lobe.

Male (sexually dimorphic characters). Based on paratype male, $7.5 \mathrm{~mm}$. Antenna 1 flagellum with strong 2-field callynophore; calceoli present. Antenna 2 flagellum elongate; calceoli present. Uropod 3 with plumose setae on each ramus.

Etymology. Named for Nicolas Baudin, captain of the French corvette Géographe which together with the Naturaliste made an extensive scientific exploration of Australia's southern coast in 1801-1802.

Remarks. Lepidepecreum baudini is one of a group of seven species in which the basis of pereopod 7 is developed beyond the merus: L. baudini; L. clypeatum Chevreux, 1888; L. clypodentatum J.L. Barnard, 1962; L. dampieri; L. flindersi; L. freycineti; and L. subclypeatum Ruffo \& Schiecke, 1977. Within this group L. baudini and L. dampieri have no anterodistal lobes on the peduncle of antenna 1. Lepidepecreum baudini differs from $L$. dampieri most noticeably in the welldeveloped mid-dorsal carina on pleonite 2 .

Distribution. Southeastern Australia, Tasman Sea; 1840$2500 \mathrm{~m}$ depth. 


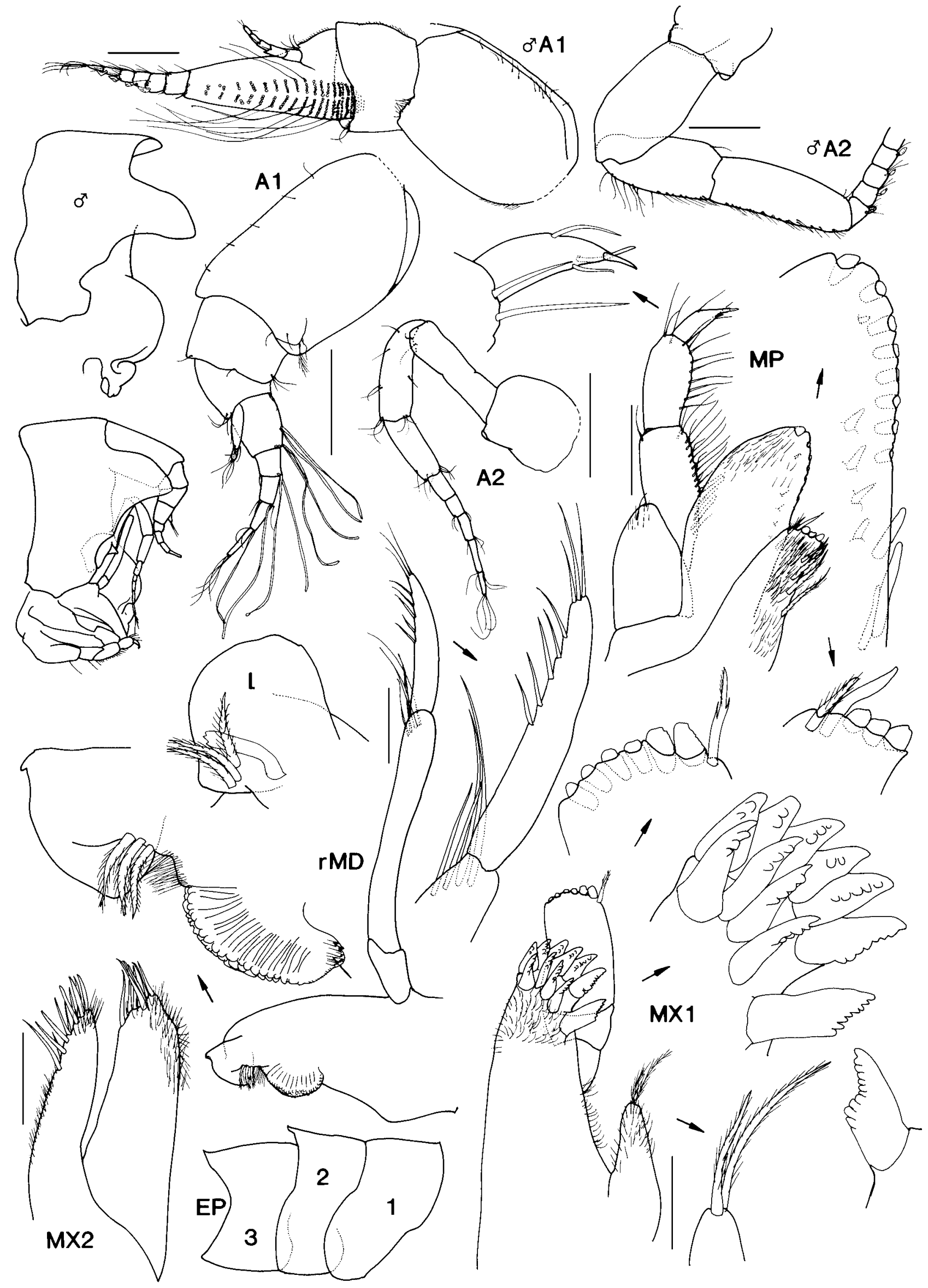

Figure 12. Lepidepecreum baudini n.sp. Holotype female, $5.5 \mathrm{~mm}$, NMV J47715; paratype male, $6.5 \mathrm{~mm}$, NMV J47717; Point Hicks, Australia. Scales for A1,2 represent $0.2 \mathrm{~mm}$, remainder represent $0.1 \mathrm{~mm}$. 


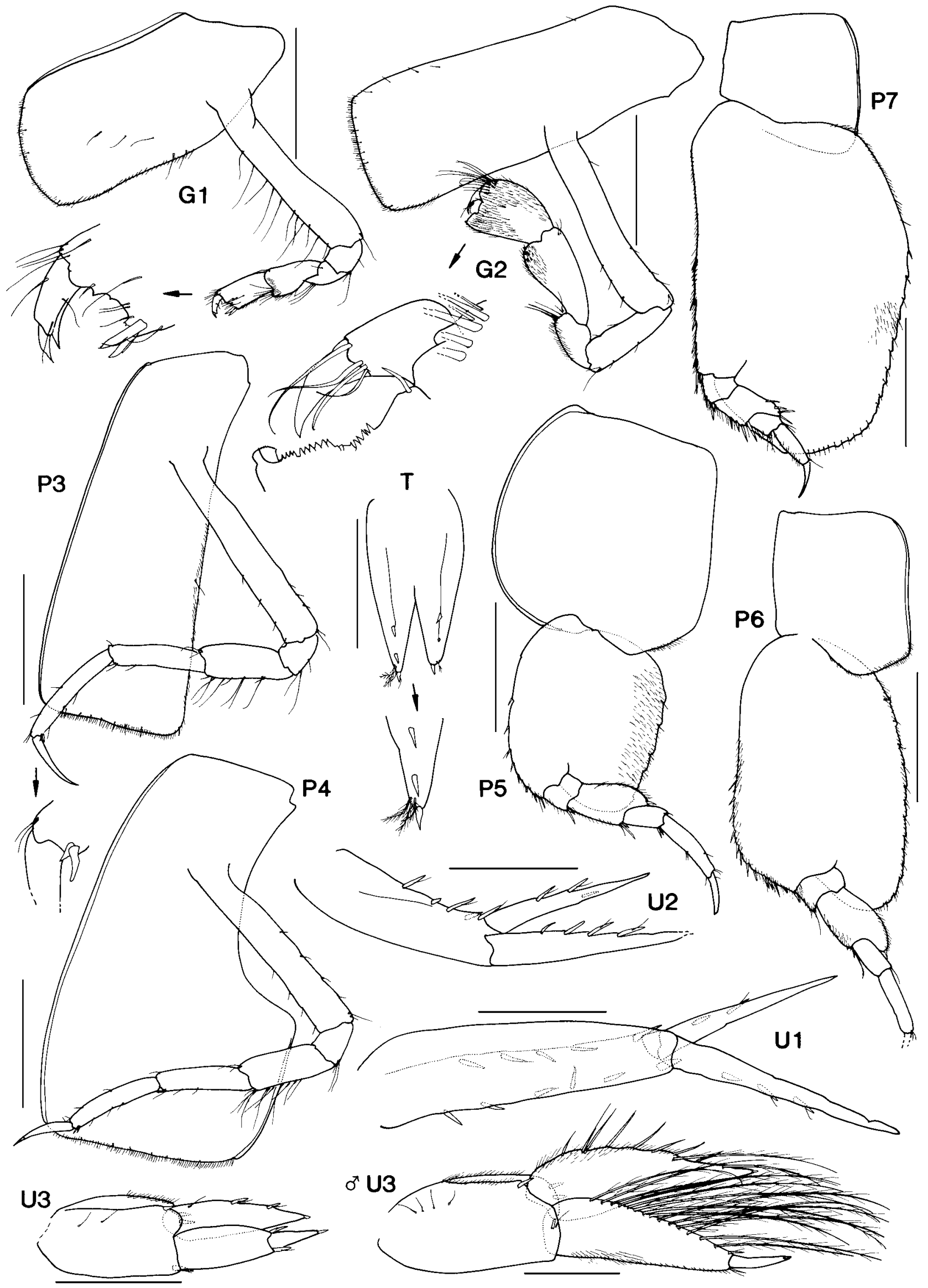

Figure 13. Lepidepecreum baudini $\mathrm{n} . \mathrm{sp}$. Holotype female, $5.5 \mathrm{~mm}$, NMV J47715; paratype male, $6.5 \mathrm{~mm}$, NMV J47717; Point Hicks, Australia. Scales for U1-3, T represent $0.1 \mathrm{~mm}$, remainder represent $0.5 \mathrm{~mm}$. 


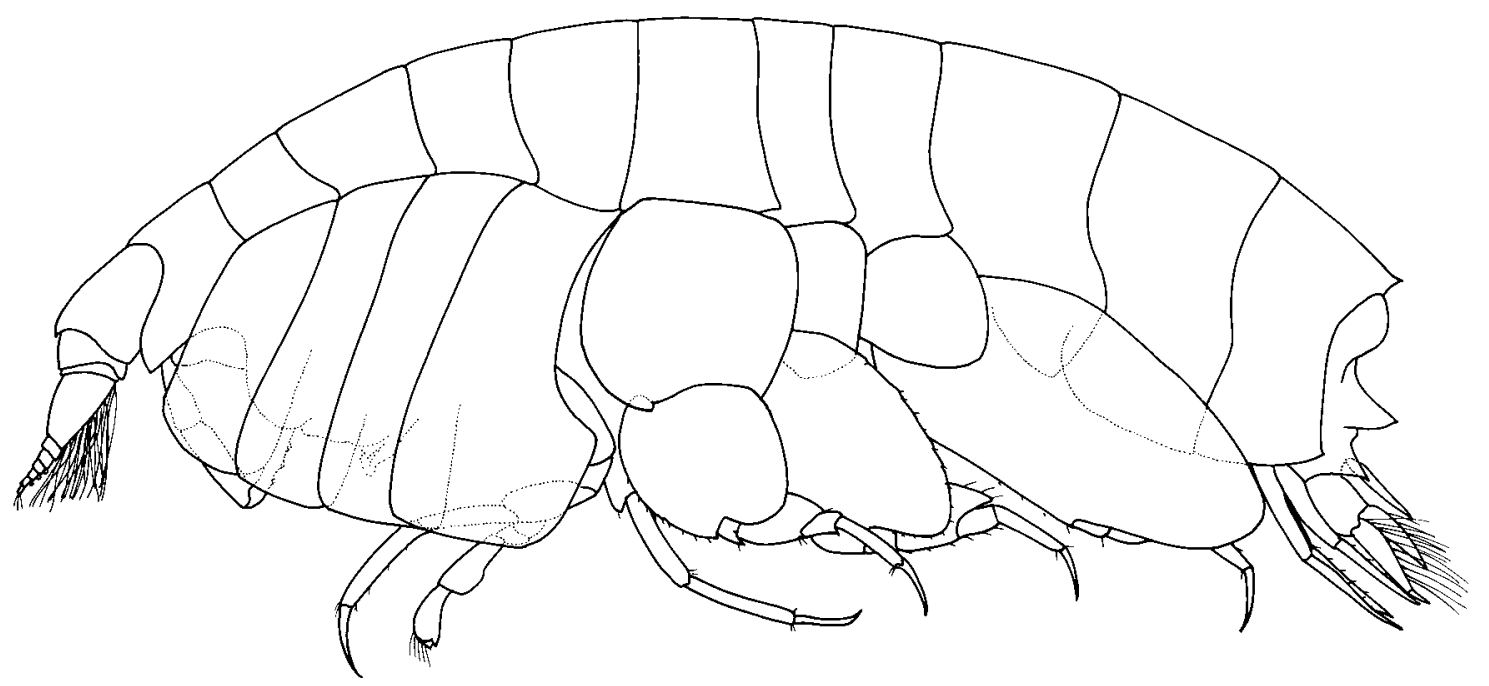

Figure 14. Lepidepecreum dampieri n.sp. Holotype male, $3.5 \mathrm{~mm}$, NMV J7670, North West Shelf, Australia.

\section{Lepidepecreum dampieri n.sp.}

Figs. 14-16

Type material. HolOTYPe, male, $3.5 \mathrm{~mm}$, NMV J7670, between Port Hedland and Dampier, North West Shelf, Western Australia, eastern Indian Ocean, $19^{\circ} 05^{\prime} 00^{\prime \prime} \mathrm{S}$ $117^{\circ} 26^{\prime} 00^{\prime \prime} \mathrm{E}$ to $19^{\circ} 05^{\prime} 00^{\prime \prime S} 117^{\circ} 46^{\prime} 23$ "E, $120-122 \mathrm{~m}$, muddy sand, dredge, 6 December 1983, G.C.B. Poore \& H.M. Lew Ton, FRV Soela stn S03/83 [NMV stn NWA-52].

Type locality. Between Port Hedland and Dampier, North West Shelf, Western Australia, eastern Indian Ocean, $19^{\circ} 05^{\prime} 00^{\prime \prime S} 117^{\circ} 26^{\prime} 00^{\prime \prime E}$ to $19^{\circ} 05^{\prime} 00^{\prime \prime S ~} 117^{\circ} 46^{\prime} 23$ "E, 120 $122 \mathrm{~m}$ depth.

Description. Based on holotype male, $3.5 \mathrm{~mm}$. Body expanded to form a lateral bulge. Head with lateral cephalic lobe a semidome, apically subacute. Antenna 1 peduncular articles 1 and 2 without anterodistal lobe; accessory flagellum 2-articulate; flagellum with strong 2-field callynophore [not known for female]; robust setae absent from proximal articles; calceoli present. Antenna 2 peduncular article 3 elongate; flagellum elongate; calceoli present. Epistome/upper lip [not known]. Mandible molar columnar with oval, fully triturating surface; mandibular palp attached proximally, article 3 without A3-setae. Maxilla 1 outer plate with setal-tooth 7 [symmetry unknown] cuspidate distally; palp distal margin with apical robust setae. Maxilliped outer plate with 1 short, slender apical robust seta.

Gnathopod 1 subchelate; coxa large, about as long as coxa 2, subrectangular with straight anterior margin; basis sparsely setose along anterior margin; ischium short; carpus long, subequal in length to propodus, without posterior lobe; propodus margins subparallel, palm acute, entire, straight. Gnathopod 2 palm slightly obtuse. Pereopod 4 coxa without distinct lateral ridge, with a well-developed posteroventral lobe. Pereonite 5 dorsally smooth. Pereopod 5 coxa without distinct lateral ridge, without umbo, basis about as long as broad. Pereopod 7 basis posterodistally produced beyond merus.

Pleonite 1 without mid-dorsal carina, not produced dorsodistally. Pleonite 2 without mid-dorsal carina, not produced dorsodistally. Pleonite 3 with mid-dorsal carina, produced dorsodistally, apically acute, posterodorsal margin produced. Epimeron 3 posterior margin smooth, posteroventral corner subquadrate. Urosomite 1 with deep notch and a narrow vertically produced acute spine. Uropod 3 inner and outer rami well developed; outer ramus article 2 short; with plumose setae on each ramus. Telson moderately cleft, with dorsal robust setae, with 1 apical robust seta on each lobe.

Etymology. Named for the English privateer William Dampier, who visited northwestern Australia in 1688 and 1699 and has been described as Australia's first naturalist.

Remarks. Lepidepecreum dampieri is also one of a group of seven species in which the basis of pereopod 7 is developed beyond the merus. Lepidepecreum dampieri is most similar to L. baudini (see L. baudini remarks).

Distribution. North West Shelf, Australia, eastern Indian Ocean; $120-122$ m depth.

\section{Lepidepecreum flindersi n.sp.}

Figs. 17-19

Type material. HOLOTYPE, female, ovigerous (4 eggs), 4.0 mm, NMV J47720; 1 PARATYPE, male, 4.8 mm, NMV J47721; 5 PARATYPES, NMV J47722; 5 PARATYPES, AM P57737; 85 $\mathrm{km}$ NE of North Point, Flinders Island, eastern Bass Strait, Australia, $39^{\circ} 02.4^{\prime} \mathrm{S} 148^{\circ} 30.6^{\prime} \mathrm{E}, 120 \mathrm{~m}$, muddy sand, dredge, R. Wilson, 15 November 1981, RV Tangaroa, cruise 81-T-1, stn BSS 169. -2 PARATYPES, NMV J47723, $100 \mathrm{~km}$ NE of North Point, Flinders Island, eastern Bass Strait, Australia, $38^{\circ} 51.8^{\prime} \mathrm{S} 148^{\circ} 26.5^{\prime} \mathrm{E}, 130 \mathrm{~m}$, fine sand, epibenthic sled, R. Wilson, 15 November 1981, RV Tangaroa, cruise 81-T-1, stn BSS 170. -2 PARATYPES, NMV J47724, S of Point Hicks, Victoria, Australia, $38^{\circ} 17.70^{\prime} \mathrm{S} 149^{\circ} 11.30^{\prime} \mathrm{E}, 400 \mathrm{~m}$, coarse sand, gravel, mud, many sponges, WHOI epibenthic sled, M.F. Gomon et al., 24 July 1986, RV Franklin, stn SLOPE 40.

Type locality. $85 \mathrm{~km}$ NE of North Point, Flinders Island, eastern Bass Strait, Australia, 3902.4'S 148 $30.6^{\circ} \mathrm{E}, 120 \mathrm{~m}$ depth. 


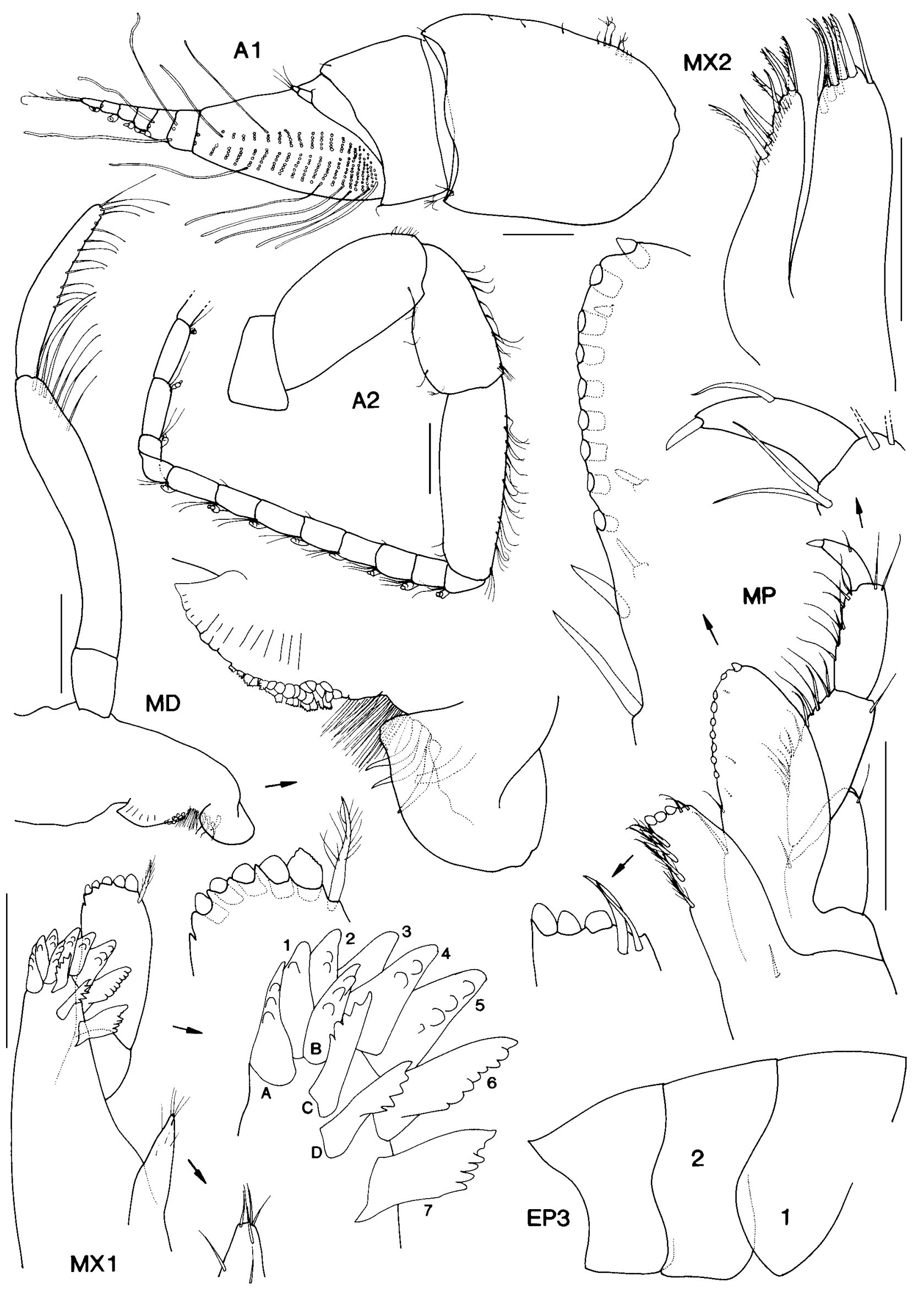

Figure 15. Lepidepecreum dampieri n.sp. Holotype male, 3.5 mm, NMV J7670, North West Shelf, Australia. Scales represent 0.1 mm. 


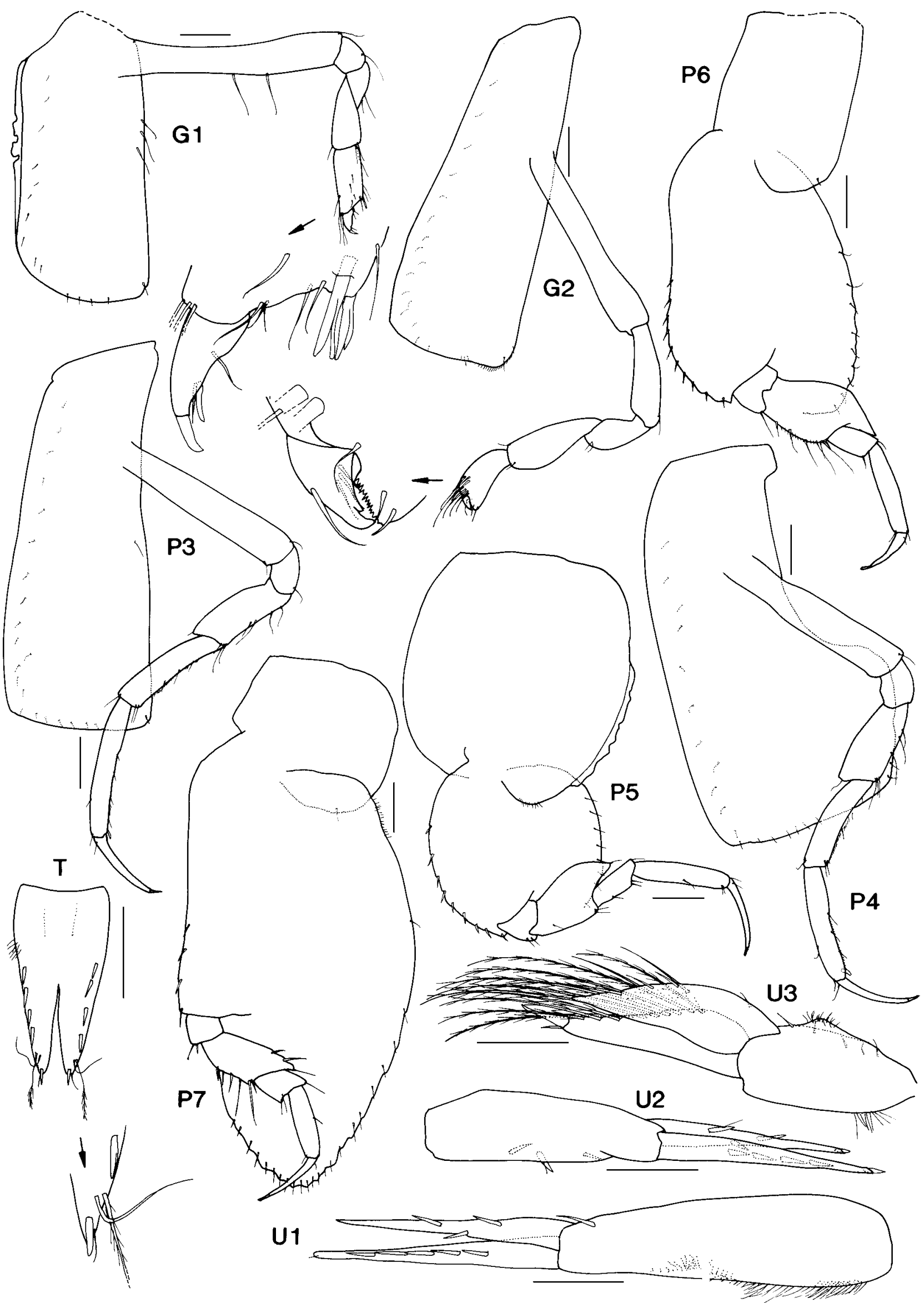

Figure 16. Lepidepecreum dampieri n.sp. Holotype male, $3.5 \mathrm{~mm}$, NMV J7670, North West Shelf, Australia. Scales represent 0.1 mm. 
Figure 17. Lepidepecreum flindersi n.sp. Paratype male, 4.8 mm, NMV J47721, Bass Strait, Australia.

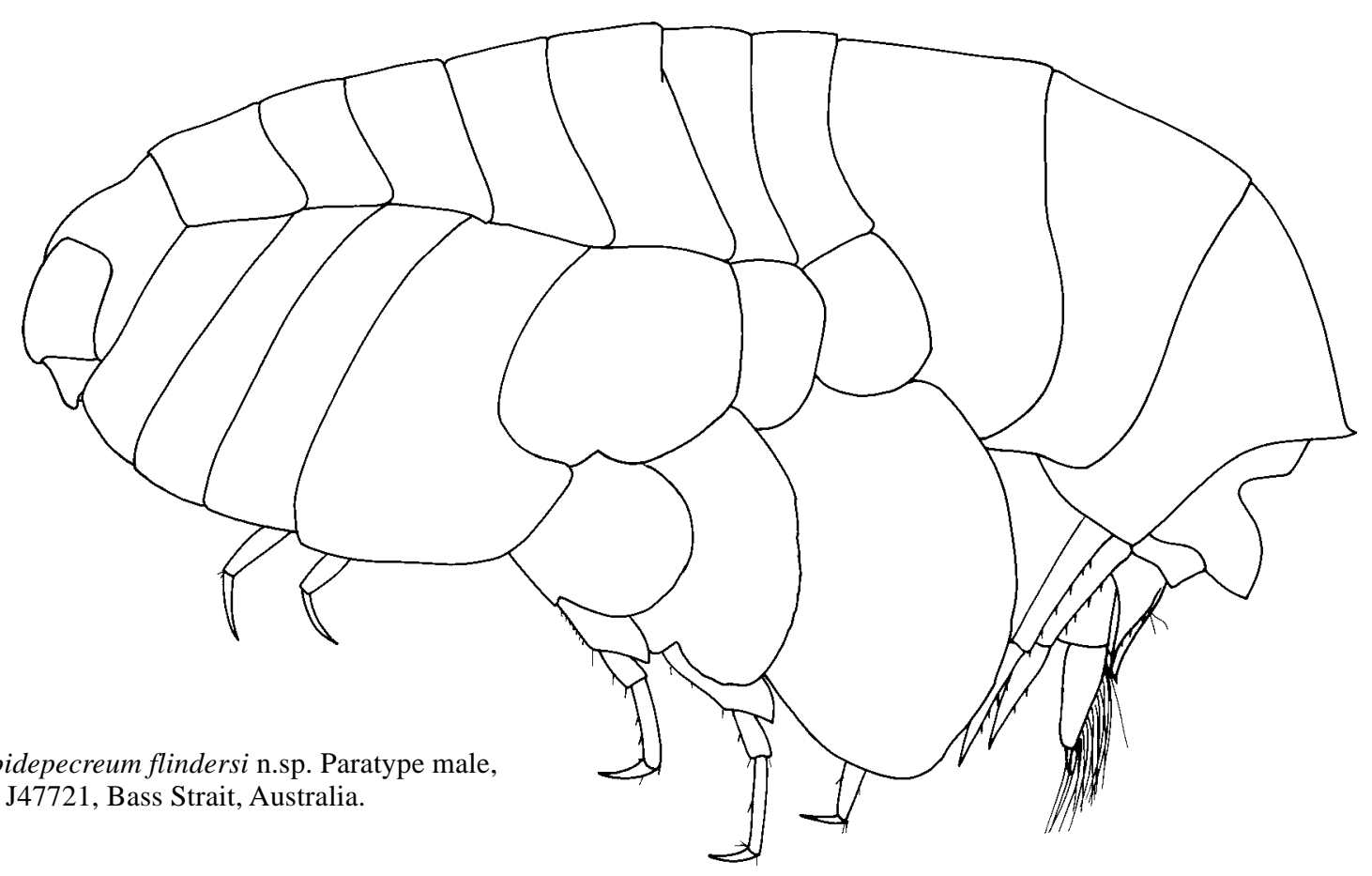

Description. Based on holotype female, $4.0 \mathrm{~mm}$. Body expanded to form a lateral bulge. Head with lateral cephalic lobe semidome, apically rounded. Antenna 1 peduncular article 1 without anterodistal lobe, peduncular article 2 with well-developed anterodistally rounded lobe; accessory flagellum 2-articulate; flagellum with weak 1-field callynophore; robust setae absent from proximal articles; calceoli absent. Antenna 2 peduncular article 3 elongate; flagellum short; calceoli absent. Epistome/upper lip with epistome produced beyond upper lip, broadly rounded; upper lip not produced. Mandible molar columnar with oval, fully triturating surface; mandibular palp attached proximally, article 3 without A3-setae. Maxilla 1 outer plate with setal-tooth 7 present, left and right asymmetrical, cuspidate distally; palp distal margin with apical robust setae. Maxilliped outer plate with 1 short, slender apical robust seta.

Gnathopod 1 subchelate; coxa large, about as long as coxa 2, subrectangular with straight anterior margin; basis sparsely setose along anterior margin; ischium short; carpus long, subequal in length to propodus, without posterior lobe; propodus with margins subparallel, palm acute, entire, straight. Gnathopod 2 palm obtuse. Pereopod 4 coxa without distinct lateral ridge, with a well-developed posteroventral lobe. Pereonite 5 dorsally smooth. Pereopod 5 coxa without distinct lateral ridge, without umbo, basis about as long as broad. Pereopod 7 basis posterodistally produced beyond merus.

Pleonite 1 without mid-dorsal carina, not produced dorsodistally. Pleonite 2 without mid-dorsal carina, not produced dorsodistally. Pleonite 3 with mid-dorsal carina, produced dorsodistally, apically acute, posterodorsal margin produced. Epimeron 3 posterior margin smooth, posteroventral corner subquadrate. Urosomite 1 without notch, with subtriangular, acutely produced boss. Uropod 3 inner and outer rami well developed; outer ramus article 2 short; without plumose setae on rami. Telson moderately cleft, without dorsal robust setae, with 1 apical robust seta on each lobe.
Male (sexually dimorphic characters). Based on paratype male, $4.8 \mathrm{~mm}$. Antenna 1 flagellum with strong 2-field callynophore; calceoli present. Antenna 2 flagellum elongate, calceoli present. Urosomite 1 with notch, with subtriangular, acutely produced boss. Uropod 3 with plumose setae on each ramus. Telson with dorsal robust setae.

Etymology. Named for Matthew Flinders, captain of the first vessel to circumnavigate Australia.

Remarks. Lepidepecreum flindersi is one of a group of seven species in which the basis of pereopod 7 is developed beyond the merus. It is most similar to the Australian species $L$. baudini and $L$. dampieri, but differs from these species in the strongly produced dorsodistal lobe on peduncular article 2 of antenna 1 and in the dorsal shape of urosomite 1 .

Distribution. Bass Strait, Australia; 120-400 m depth.

\section{Lepidepecreum foraminiferum Stebbing}

Lepidepecreum foraminiferum Stebbing, 1888: 686, pl. 24.Stebbing, 1906: 79.-J.L. Barnard, 1958: 93.-Thurston \& Allen, 1969: 361.-Mills, 1972: 74, table 1.-Lowry \& Bullock, 1976: 94.-Barnard \& Karaman, 1991: 496.-De Broyer \& Jazdzewski, 1993: 70.

Anonyx longicornis.-Della Valle, 1893: 814 (in part).

Lepidepecreum forameniferum [sic].-Gurjanova, 1962: 324 (key). not Lepidepecreum foraminiferum.-Nayar, 1959: 7, pl. 1 figs. 16-26.-Nayar, 1966: 136.-Surya Rao, 1972: 199 (= Lepidepecreum sp.).

Material examined. Two specimens, NMV J47725, off Enderby Land, Antarctica, Southern Ocean, 65 $56.40^{\prime} \mathrm{S}$ $50^{\circ} 52.10^{\prime} \mathrm{E}, 386-400 \mathrm{~m}$, shelly mud, silt and bryozoan shell, WHOI epibenthic sled, M. Norman, 15 November 1985, MS Nella Dan, stn HRD 010. - 3 females, NMV J38196, western part of MacRobertson Shelf, Antarctica, 66 53.69'S $63^{\circ} 06.34^{\prime} \mathrm{E}$ to $66^{\circ} 53.67^{\prime} \mathrm{S} 63^{\circ} 05.51^{\prime} \mathrm{E}, 367-439 \mathrm{~m}$, epibenthic sled, M. O'Loughlin on RSV Aurora Australis, 


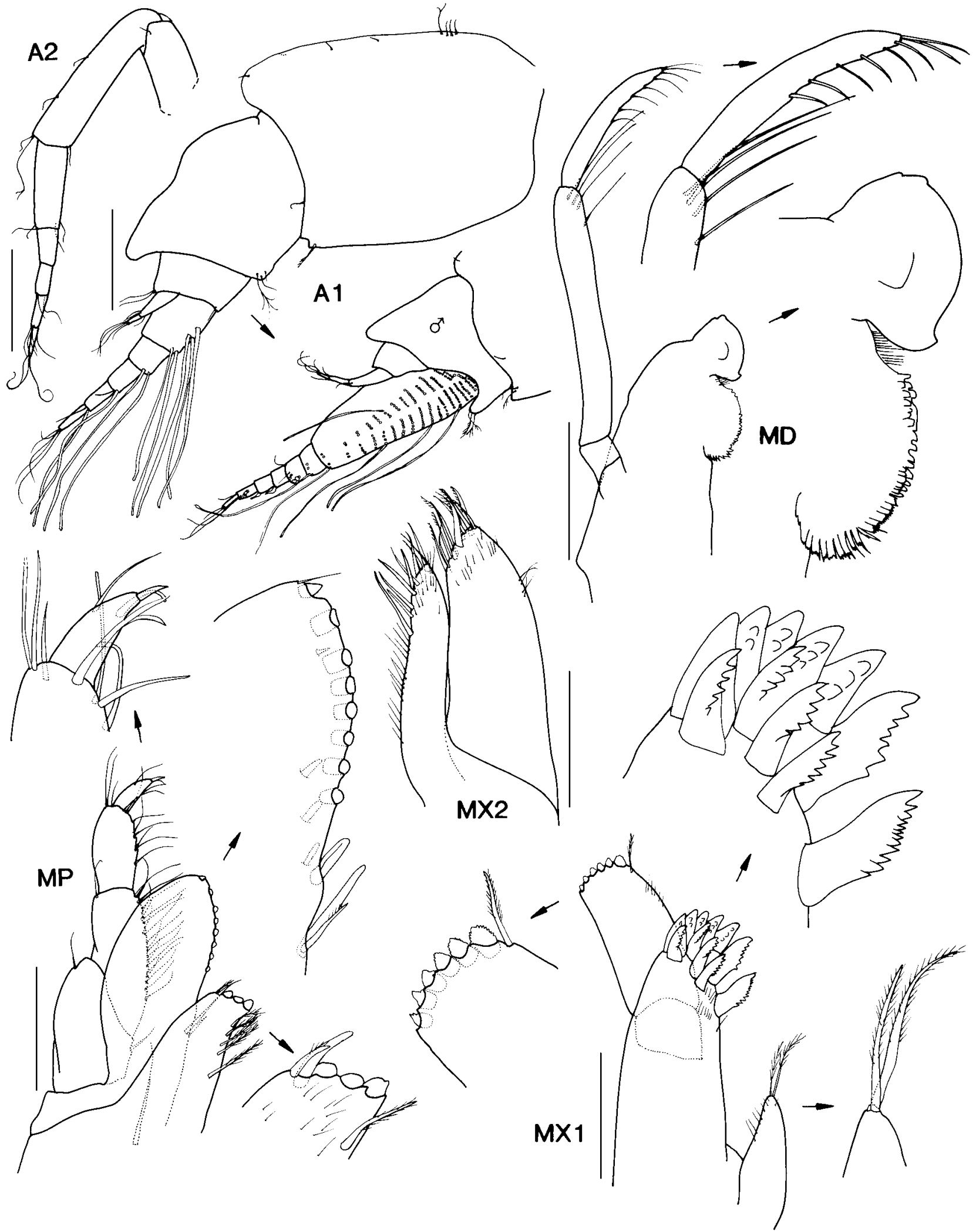

Figure 18. Lepidepecreum flindersi n.sp. Holotype female, 4.0 mm, NMV J47720; paratype male, 4.8 mm, NMV J47721; Bass Strait, Australia.

11 February 1993, stn AA93-125. — 5 specimens (females and immature), NMV J38195, off the Larsemann Hills, eastern Prydz Bay, Antarctica, 68 $54.88^{\prime} \mathrm{S} 76^{\circ} 37.03^{\prime} \mathrm{E}$ to $68^{\circ} 54.82^{\prime} \mathrm{S} 76^{\circ} 37.71^{\prime} \mathrm{E}, 667-716 \mathrm{~m}$, epibenthic sled, 18 February 1993, M. O'Loughlin on RSV Aurora Australis, stn AA93-158.
Type locality. Off Cumberland Bay, Kerguelen Island,

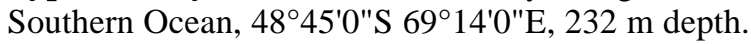

Remarks. The material from India described as $L$. foraminiferum by Nayar (1959) is distinguished from this species by the absence of the strongly produced anterodistal 


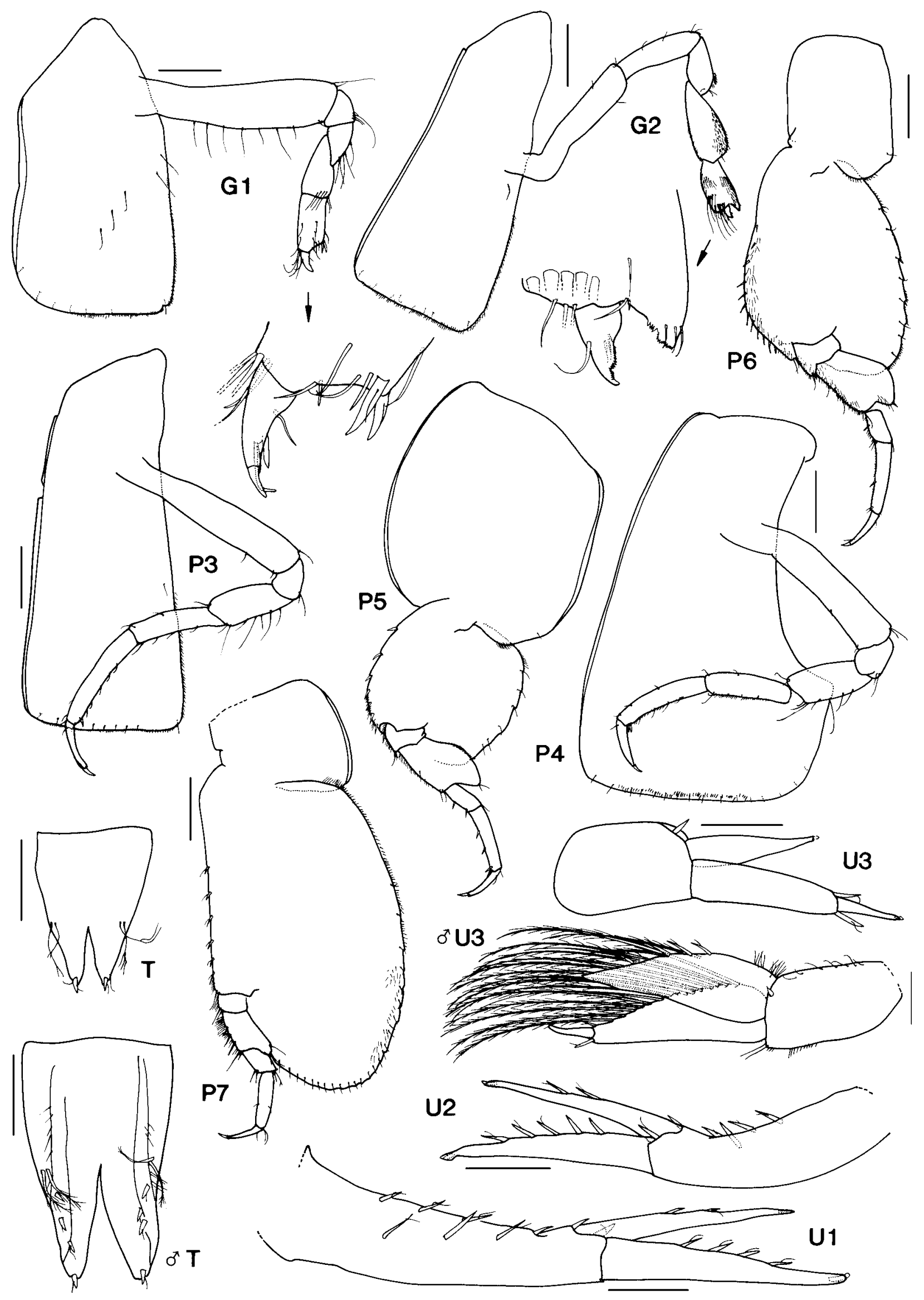

Figure 19. Lepidepecreum flindersi n.sp. Holotype female, 4.0 mm, NMV J47720; paratype male, 4.8 mm, NMV J47721; Bass Strait, Australia. 
lobe on antenna 1 peduncular article 1 ; and by the shape of pereopod 7 merus, which has a strong posteroproximal shoulder.

The specimens reported here are the first records of $L$. foraminiferum since its original description from Kerguelen Island, and extend the species distribution from the subantarctic to the Antarctic continent.

Distribution. Enderby Land, MacRobertson Shelf and Prydz Bay, Antarctica; Kerguelen Island, Southern Ocean; 232-716 m depth.

\section{Lepidepecreum freycineti n.sp.}

Figs. 20-22

Type material. HolOTYPE, female, ovigerous (8 eggs), 4.0 mm, NMV J47726; 4 PARATYPES, NMV J47727; 5 PARATYPES, AM P57738; off Freycinet Peninsula, Tasmania, Australia, $42^{\circ} 0.20^{\prime} \mathrm{S} 148^{\circ} 37.70^{\prime} \mathrm{E}, 720 \mathrm{~m}$, coarse shelly sand, WHOI epibenthic sled, M.F. Gomon et al., 27 July 1986, RV Franklin, stn SLOPE 46. - 1 PARATYPE, NMV J47728, S of Point Hicks, Victoria, Australia, 38 $19.10^{\prime} \mathrm{S} 149^{\circ} 14.30^{\prime} \mathrm{E}, 600 \mathrm{~m}$, coarse sand, WHOI epibenthic sled, M.F. Gomon et al., 24 July 1986 , RV Franklin stn SLOPE 39. - 1 PARATYPE, NMV J47729, off Freycinet Peninsula, Tasmania, Australia, $42^{\circ} 2.20^{\prime} \mathrm{S}$ $148^{\circ} 38.70^{\prime} \mathrm{E}, 800 \mathrm{~m}$, coarse shelly sand, WHOI epibenthic sled, M.F. Gomon et al., 27 July 1986, RV Franklin, stn SLOPE 45.

Type locality. Off Freycinet Peninsula, Tasmania, Australia, $42^{\circ} 0.20^{\prime} \mathrm{S} 148^{\circ} 37.70^{\prime} \mathrm{E}, 720 \mathrm{~m}$ depth.

Description. Based on holotype female, $4.0 \mathrm{~mm}$. Body expanded to form a lateral bulge. Head with lateral cephalic lobe semidome, apically subacute. Antenna 1 peduncular article 1 with well-developed anterodistally subacute lobe; article 2 without anterodistal lobe; accessory flagellum 2articulate; flagellum with weak 1-field callynophore; robust setae absent from proximal articles; calceoli absent. Antenna 2 peduncular article 3 elongate; flagellum short, calceoli absent. Epistome/upper lip with epistome produced beyond upper lip (slightly), broadly rounded. Mandible molar columnar with oval, fully triturating surface; mandibular palp attached proximally, article 3 without A3-setae. Maxilla 1 outer plate with left and right setal-tooth 7 asymmetrical, cuspidate distally; palp distal margin with apical robust setae. Maxilliped outer plate with 1 short, slender apical robust seta.

Gnathopod 1 subchelate; coxa large, about as long as coxa 2, subrectangular with straight anterior margin; basis sparsely setose along anterior margin; ischium short; carpus long, subequal in length to propodus, without posterior lobe; propodus margins subparallel, palm acute, entire, straight. Gnathopod 2 palm obtuse (concave). Pereopod 4 coxa without distinct lateral ridge, with a well-developed posteroventral lobe. Pereonite 5 dorsally smooth. Pereopod 5 coxa without distinct lateral ridge, without umbo, basis about as long as broad. Pereopod 7 basis posterodistally produced beyond merus.

Pleonite 1 without mid-dorsal carina, not produced dorsodistally. Pleonite 2 without mid-dorsal carina, not produced dorsodistally. Pleonite 3 with mid-dorsal carina, produced dorsodistally, apically acute, posterodorsal margin not produced. Epimeron 3 posterior margin smooth, posteroventral corner narrowly rounded. Urosomite 1 projecting over urosomite 2 , with a narrow vertically produced recurved acute spine. Uropod 3 inner ramus significantly reduced; outer ramus article 2 long; without plumose setae on rami. Telson moderately cleft, with dorsal robust setae, with 1 apical robust seta on each lobe.

Etymology. Named for Louis de Freycinet, LieutenantCommander and cartographer on the French corvette Naturaliste and later Commander of the schooner Casuarina which accompanied the Géographe on the latter part of Baudin's voyage around the Australian coasts in 1802-1803.

Remarks. Lepidepecreum freycineti is also one of a group of seven species in which the basis of pereopod 7 is developed beyond the merus. It is similar to L. clypeatum, L. clypodentatum and L. subclypeatum in having a anterodistal lobe on peduncular article 1 of antenna 1. Lepidepecreum freycineti and L. clypodentatum are the only two species of this group without an anterodistal lobe on article 2 . These two species are easily distinguished by the dorsodistal projections on pleonites 1 and 2 in $L$. clypodentatum.

Distribution. Southeastern Australia, Tasman Sea; 600-800 $\mathrm{m}$ depth.

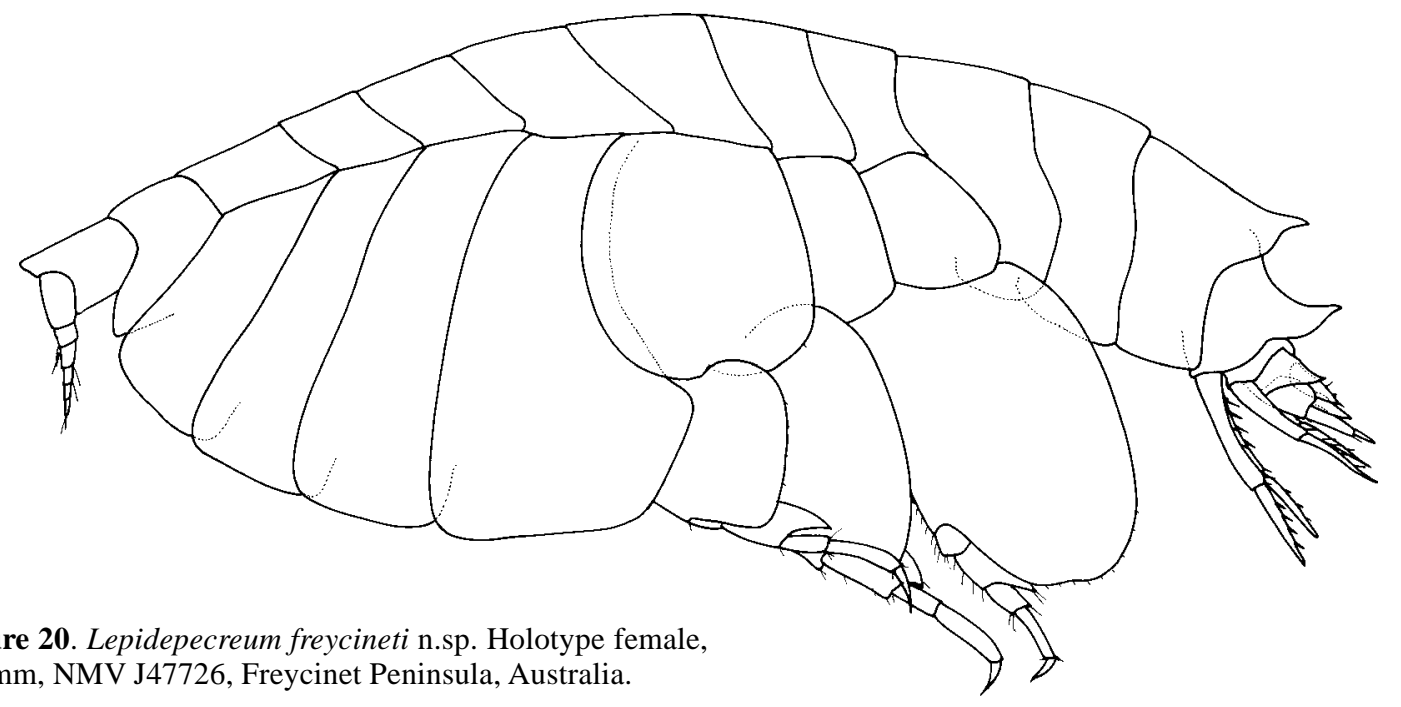

4.0 mm, NMV J47726, Freycinet Peninsula, Australia. 


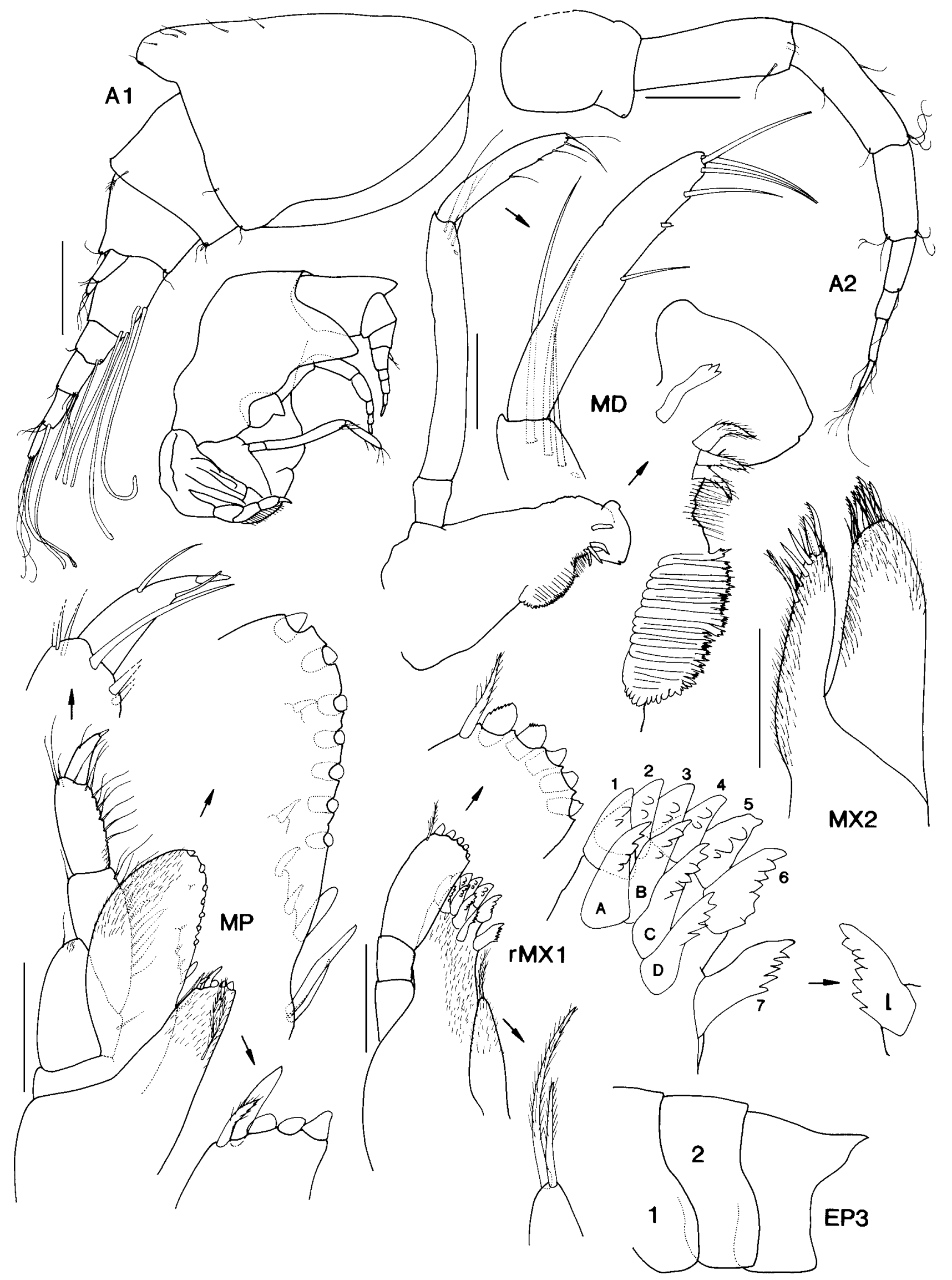

Figure 21. Lepidepecreum freycineti n.sp. Holotype female, $4.0 \mathrm{~mm}$, NMV J47726, Freycinet Peninsula, Australia. Scales represent $0.1 \mathrm{~mm}$ 


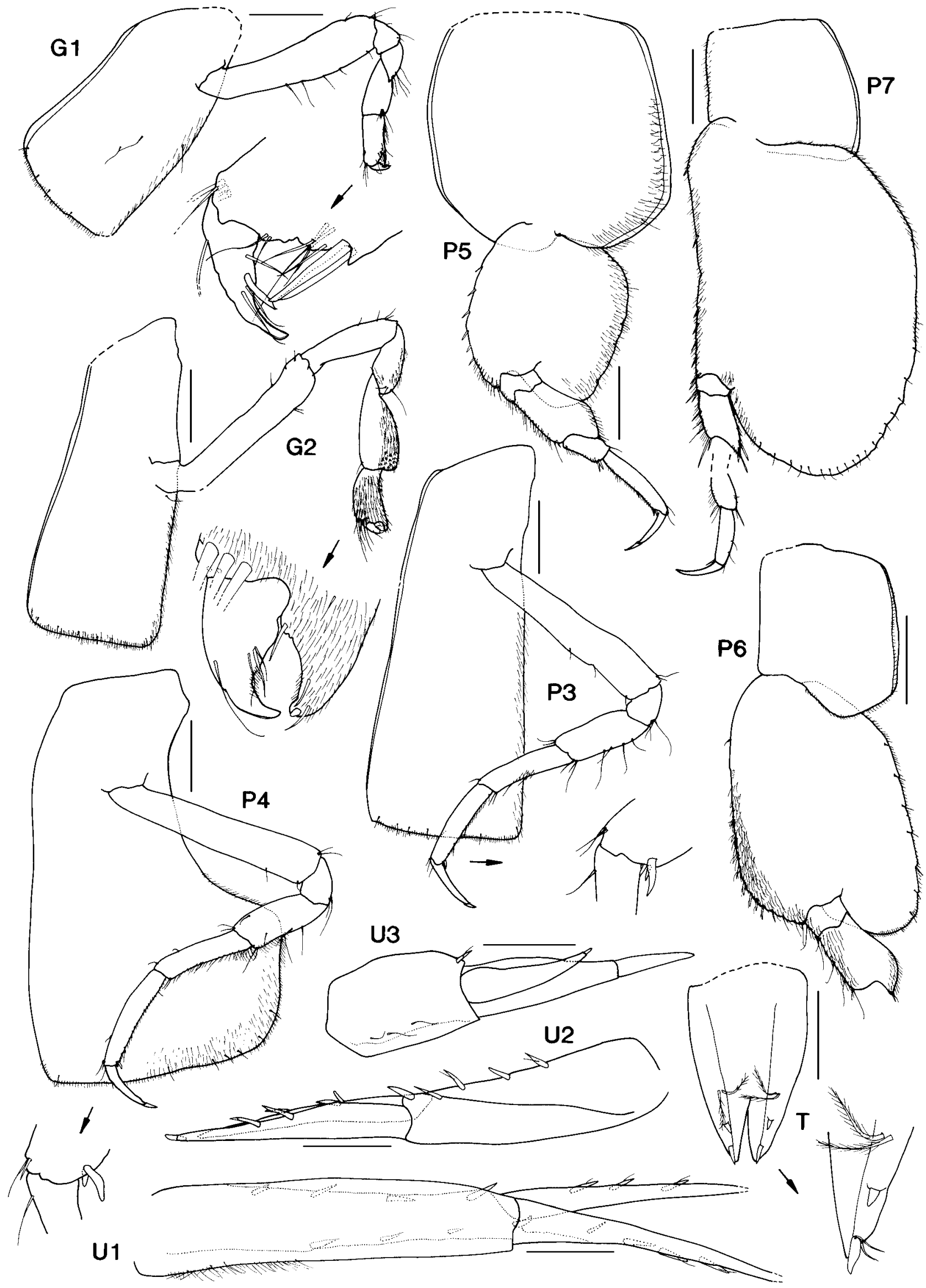

Figure 22. Lepidepecreum freycineti n.sp. Holotype female, $4.0 \mathrm{~mm}$, NMV J47726, Freycinet Peninsula, Australia. Scales for U1-3, T represent $0.1 \mathrm{~mm}$, remainder represent $0.2 \mathrm{~mm}$. 


\section{Lepidepecreum infissum Andres}

Lepidepecreum infissum Andres, 1983: 191, fig. 3.-Andres, 1985 : 134.-Barnard \& Karaman, 1991: 496.-De Broyer \& Jazdzewski, 1993: 70.

Material examined. One specimen, NMV J38269, southern part of Amery Depression, Antarctica, 68 $59.20^{\prime} \mathrm{S} 74^{\circ} 59.56^{\prime} \mathrm{E}$, 746 m, diatomaceous ooze, Van Veen grab, 22 January 1993, M. O'Loughlin on RSV Aurora Australis, stn AA93-37. - 23 specimens, NMV J38270, western part of MacRobertson Shelf, Antarctica, $66^{\circ} 55.51^{\prime} \mathrm{S} 62^{\circ} 32.72^{\prime} \mathrm{E}$ to $66^{\circ} 55.33^{\prime} \mathrm{S} 62^{\circ} 32.16^{\prime} \mathrm{E}$, 113 m, epibenthic sled, 11 February 1993, M. O'Loughlin on RSV Aurora Australis, stn AA93-124. - 13 specimens, NMV J38271, N end of Fram Bank, Antarctica, 67 05.03 'S $68^{\circ} 58.80^{\prime} \mathrm{E}$ to $67^{\circ} 05.24^{\prime} \mathrm{S} 68^{\circ} 58.51^{\prime} \mathrm{E}, 204-216 \mathrm{~m}$, WHOI epibenthic sled, 13 February 1993, M. O'Loughlin on RSV Aurora Australis, stn AA93-131. - 10 specimens, NMV J38272, Amery Depression, Antarctica, 68 ${ }^{\circ} 04.366^{\prime} \mathrm{S} 72^{\circ} 17.81^{\prime} \mathrm{E}$ to $68^{\circ} 04.44^{\prime} \mathrm{S} 72^{\circ} 18.11^{\prime} \mathrm{E}, 765 \mathrm{~m}$, epibenthic sled, 17 February 1993, M. O'Loughlin on RSV Aurora Australis, stn AA93151. —5 specimens, NMV J38273, Amery Depression, Antarctica, $68^{\circ} 26.47^{\prime} \mathrm{S} 72^{\circ} 17.81^{\prime} \mathrm{E}$ to $68^{\circ} 54.82^{\prime} \mathrm{S} 73^{\circ} 37.53^{\prime} \mathrm{E}$, 765 m, epibenthic sled, 17 February 1993, M. O'Loughlin on RSV Aurora Australis, stn AA93-154. -1 specimen, NMV J38274, off the Larsemann Hills, eastern Prydz Bay, Antarctica, $68^{\circ} 54.88^{\prime} \mathrm{S} 76^{\circ} 37.03^{\prime} \mathrm{E}$ to $68^{\circ} 54.82$ 'S 76³7.71'E, 667-716 m, epibenthic sled, 18 February 1993, M. O'Loughlin on RSV Aurora Australis, stn AA93-158.

Type locality. Antarctic Peninsula, $63^{\circ} 22^{\prime} \mathrm{S} 54^{\circ} 10^{\prime} \mathrm{W}, 0$ $223 \mathrm{~m}$ over water depth $235 \mathrm{~m}$.

Remarks. These records extend the known distribution of L. infissum into the eastern Antarctic.

Distribution. Antarctic Peninsula, Amery Depression, MacRobertson Shelf and Prydz Bay, Antarctica; 113-765 m depth.

\section{Lepidepecreum tourville n.sp.}

Figs. 23-25

Type material. HOLOTYPE, female, ovigerous (9 eggs), 5.8 mm, NMV J47730; 1 PARATYPE, male, $5.6 \mathrm{~mm}$, NMV J17159; 3 PARATYPES, (2 ovigerous females, 1 immature), AM P57739; 48 km ENE Cape Tourville, Tasmania, Australia, $42^{\circ} 00.25^{\prime} \mathrm{S} 148^{\circ} 43.55^{\prime} \mathrm{E}$ to $41^{\circ} 57.77$ 'S $148^{\circ} 42.08^{\prime} \mathrm{E}, 1264$ $1130 \mathrm{~m}$, gravel with lumps of sandy mud aggregate, WHOI epibenthic sled, G.C.B. Poore et al., 30 October 1988, RV Franklin stn SLOPE 81. - 1 PARATYPE, immature, NMV J47731, S of Point Hicks, Victoria, Australia, 38 $25.90^{\prime}$ S $148^{\circ} 58.60^{\prime} \mathrm{E}, 1850 \mathrm{~m}$, muddy, sandstone, WHOI epibenthic sled, G.C.B. Poore et al., 22 July 1986, RV Franklin stn SLOPE 25. - 1 PARATYPE, ovigerous female, NMV J47732, S of Point Hicks, Victoria, Australia, $38^{\circ} 25.00^{\prime} \mathrm{S} 149^{\circ} 0.00^{\prime} \mathrm{E}$, $1500 \mathrm{~m}$, compacted clay, WHOI epibenthic sled, G.C.B. Poore et al., 22 July 1986, RV Franklin, stn SLOPE 27. 1 PARATYPE, female, NMV J47733, ESE of Nowra, NSW, Australia, $54 \mathrm{~km} 34^{\circ} 52.72 ' \mathrm{~S} 151^{\circ} 15.04^{\prime} \mathrm{E}, 996 \mathrm{~m}$, mud, fine sand, fine shell, WHOI epibenthic sled, G.C.B. Poore et al., 22 October 1988, RV Franklin stn SLOPE 53.

Type locality. $48 \mathrm{~km}$ ENE of Cape Tourville, Tasmania, Australia, $42^{\circ} 00.25^{\prime} \mathrm{S} 148^{\circ} 43.55^{\prime} \mathrm{E}$ to $41^{\circ} 57.77^{\prime} \mathrm{S} 148^{\circ} 42.08^{\prime} \mathrm{E}$, 1264-1130 m depth.

Description. Based on holotype female, $5.8 \mathrm{~mm}$. Body expanded to form a lateral bulge. Head with lateral cephalic lobe semidome, apically subacute. Antenna 1 peduncular article 1 with well-developed anterodistally subacute lobe; article 2 without anterodistal lobe; accessory flagellum 2articulate; flagellum with weak 1-field callynophore; robust setae absent from proximal articles; calceoli absent. Antenna 2 peduncular article 3 elongate; flagellum short, calceoli absent. Epistome/upper lip with epistome produced beyond upper lip, broadly rounded. Mandible molar columnar with oval, fully triturating surface; mandibular palp attached proximally, article 3 without proximal A3-setae. Maxilla 1

Figure 23. Lepidepecreum tourville n.sp. Holotype female, 5.8 mm, NMV J47730, Cape Tourville, Australia.

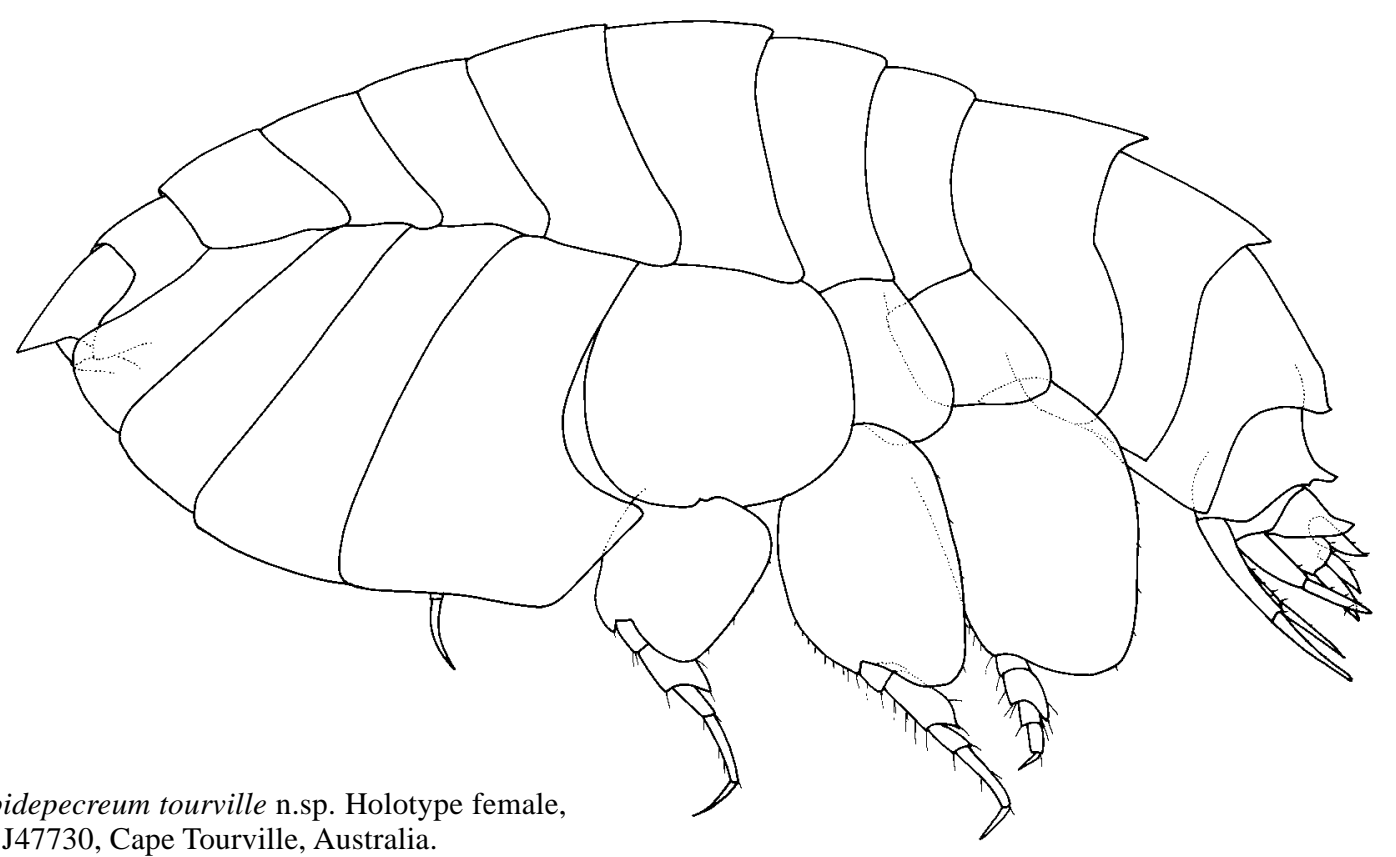




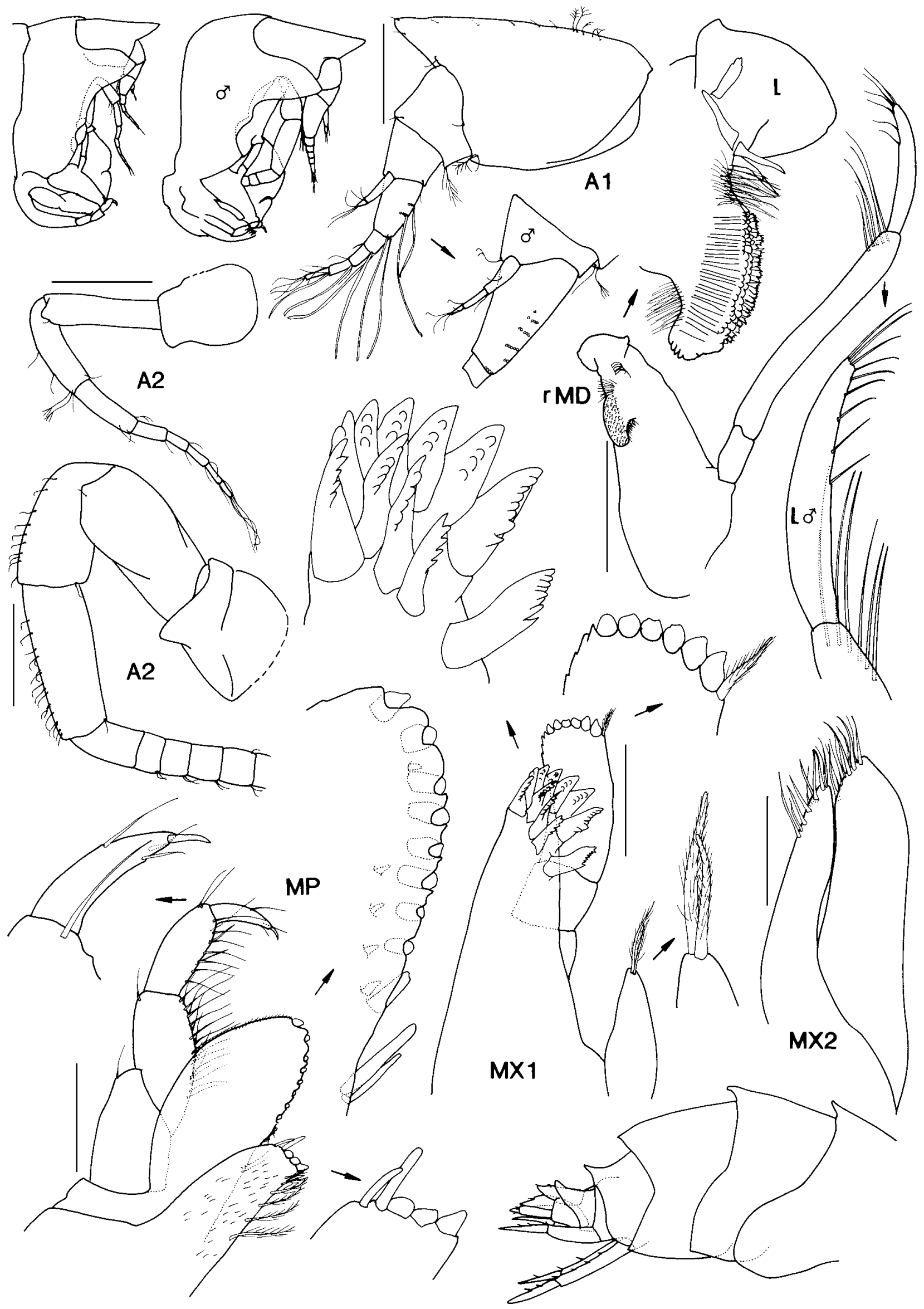

Figure 24. Lepidepecreum tourville n.sp. Holotype female, $5.8 \mathrm{~mm}$, NMV J47730; paratype male, 5.6 mm, NMV J17159; Cape Tourville, Australia. Scales represent $0.2 \mathrm{~mm}$. 


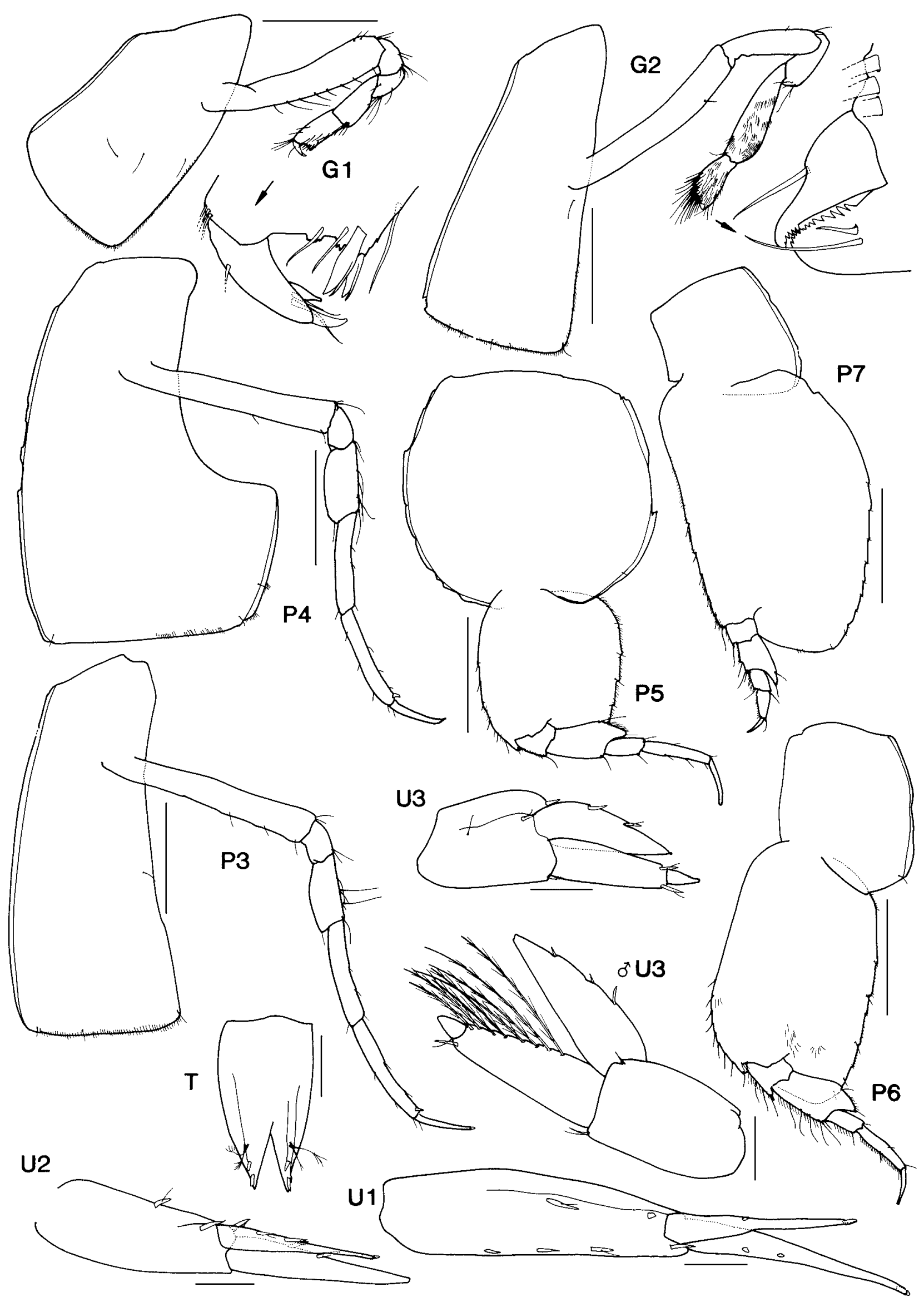

Figure 25. Lepidepecreum tourville n.sp. Holotype female, $5.8 \mathrm{~mm}$, NMV J47730; paratype male, $5.6 \mathrm{~mm}$, NMV J17159; Cape Tourville, Australia. Scales for U1-3 represent $0.1 \mathrm{~mm}$, remainder represent $0.5 \mathrm{~mm}$. 
outer plate with left and right setal-tooth 7 asymmetrical, cuspidate distally; palp distal margin with apical robust setae. Maxilliped outer plate with 1 short, slender apical robust seta.

Gnathopod 1 subchelate; coxa large, about as long as coxa 2, subrectangular with straight anterior margin; basis moderately setose along anterior margin; ischium short; carpus long, subequal in length to propodus, without posterior lobe; propodus with margins subparallel, palm acute, entire, straight. Gnathopod 2 palm obtuse. Pereopod 4 coxa without distinct lateral ridge, with a well-developed posteroventral lobe. Pereonite 5 dorsally smooth. Pereopod 5 coxa without distinct lateral ridge, without umbo, basis about as long as broad. Pereopod 7 basis posterodistally produced less than halfway along merus.

Pleonite 1 with mid-dorsal carina, acutely produced posterodistally. Pleonite 2 with mid-dorsal carina, acutely produced posterodistally. Pleonite 3 with mid-dorsal carina, produced dorsodistally, apically acute, posterodorsal margin produced. Epimeron 3 posterior margin smooth, posteroventral corner subquadrate. Urosomite 1 projecting over urosomite 2, dorsodistally acute. Uropod 3 inner and outer rami well developed; outer ramus article 2 short; without plumose setae on rami. Telson moderately or slightly cleft, with dorsal robust setae, with 1 apical robust seta on each lobe.

Male (sexually dimorphic characters). Based on paratype male, $5.6 \mathrm{~mm}$. Antenna 2 flagellum elongate, calceoli absent. Uropod 3 with plumose setae on each ramus.

The only male specimen available for this species is not fully mature; although antenna 2 is long, another moult can be seen inside. The final male stage may develop calceoli.

Etymology. Named for Cape Tourville, near the type locality.

Remarks. Lepidepecreum tourville is the only Australian species of Lepidepecreum without a greatly produced basis on pereopod 7. However it does have an anterodistal lobe on peduncular article 1 , but not on article 2 and it has dorsodistally produced pleonites 1 to 3 , a combination of characters which also occurs in the group of taxa with a large basis on pereopod 7. In fact $L$. tourville appears to be most similar to L. clypodentatum. They differ mainly in the dorsodistal lobe of antenna 1 , which is more rounded in $L$. clypodentatum, and in the development of the basis of pereopod 7 .

Distribution. Southeastern Australia, Tasman Sea; 996$1850 \mathrm{~m}$ depth.

\section{Lepidepecreum urometacarinatum Andres}

Lepidepecreum carinatum Andres, 1983: 187, figs. 1, 2 [not $L$. carinatum Bate \& Westwood, 1861].

Lepidepecreum urometacarinatum Andres, 1985: 134 [new name for L. carinatum Andres, 1983].-Barnard \& Karaman, 1991: 496.-De Broyer \& Jazdzewski, 1993: 70.

Material examined. 723 specimens (males, females and juveniles), USNM 306583; 50 specimens, AM P57740; Weddell Sea, Antarctica, $74^{\circ} 07^{\prime} \mathrm{S} 39^{\circ} 38^{\prime} \mathrm{W}, 731 \mathrm{~m}$, epibenthic sled, 6 February 1968, USCGC Glacier, IWSOE cruise 1, stn 0001. — 1 specimen, NMV J12923, off Enderby
Land, Antarctica, Southern Ocean, 66 $2.90^{\prime} \mathrm{S} 49^{\circ} 49.40^{\prime} \mathrm{E}$ to $66^{\circ} 3.00^{\prime} \mathrm{S} 49^{\circ} 49.40^{\prime} \mathrm{E}, 738$ OR $806 \mathrm{~m}$, epibenthic sled, M. Norman, MS Nella Dan stn HRD 012.

Type locality. Antarctic Peninsula, $64^{\circ} 00^{\prime} \mathrm{S} 64^{\circ} 30^{\prime} \mathrm{W}, 100-$ $382 \mathrm{~m}$ over water depth $385 \mathrm{~m}$.

Remarks. These records extend the known distribution of L. urometacarinatum into the eastern Antarctic.

Distribution. Antarctic Peninsula, Weddell Sea and Enderby Land, Antarctica; 100-806 m depth.

ACKNOWLEDGMENTS. We thank the late Eric Dahl, who originally made the LUCE lysianassoid amphipods available to us, Gary Poore (NMV), and Rafael Lemaitre (then of SOSC) for making other material available to us; Rachael Peart and Kate Dempsey for the illustrations; and Roger Springthorpe for making the plates.

\section{References}

Andres, H.G., 1983. Die Gammaridea (Crustacea: Amphipoda) der Deutschen Antarktis-Expeditionen 1975/76 und 1977/78. 3. Lysianassidae. Mitteilungen aus dem Hamburgischen Zoologischen Museum und Institut 80: 183-220.

Andres, H.G., 1985. Die Gammaridea (Crustacea: Amphipoda) der Deutschen Antarktis-Expeditionen 1975/76 und 1977/78. 4. Acanthonotozomatidae, Paramphithoidae und Stegocephalidae. Mitteilungen aus dem Hamburgischen Zoologischen Museum und Institut 82: 119-153.

Austin, W.C., 1985. An Annotated Checklist of Marine Invertebrates in the Cold Temperate Northeast Pacific. Volume 3. Cowichan, B.C.: Khoyatan Marine Laboratory, 682 pp.

Barnard, J.L., 1958. Index to the families, genera, and species of the gammaridean Amphipoda (Crustacea). Allan Hancock Foundation Publications, Occasional Paper 19: 1-145.

Barnard, J.L., 1962. South Atlantic abyssal amphipods collected by R.V. Vema. Abyssal Crustacea. Vema Research Series 1: 178.

Barnard, J.L., 1969. The families and genera of marine gammaridean Amphipoda. United States National Museum Bulletin 271: 1-535.

Barnard, J.L., 1971. Gammaridean Amphipoda from a deepsea transect off Oregon. Smithsonian Contributions to Zoology 61: 1-86.

Barnard, J.L. \& G.S. Karaman, 1991. The families and genera of marine gammaridean Amphipoda (except marine gammaroids). Records of the Australian Museum, Supplement 13(2): 419-866.

Barnard, K.H., 1931. Diagnosis of new genera and species of amphipod Crustacea collected during the "Discovery" investigations, 1925-1927. Annals and Magazine of Natural History, Series 10, 7: 425-430.

Barnard, K.H., 1932. Amphipoda. Discovery Reports 5: 1-326, pl. 1.

Bate, C.S., 1862. Catalogue of the Specimens of Amphipodous Crustacea in the Collection of the British Museum. London: Trustees, British Museum, 399 pp., 58 pls.

Bate, C.S., \& J.O. Westwood, 1861. A History of the British Sessile-eyed Crustacea. Vol. I, pp. 1-144. London: John Van Voorst.

Brattström, H., \& E. Dahl, 1951. Reports of the Lund University Chile Expedition 1948-49. 1. General account, lists of stations, hydrography. Lunds Universitets Årsskrift N.F. Avd. 2 46(8): 1-88

Budnikova, L.L., 1999. Two new lysianassid species (Amphipoda, Gammaridea) from coastal waters of West Sakhalin, Sea of Japan. Publications of the Seto Marine Biological Laboratory 38(5/6): 237-253. 
Chevreux, E., 1888. Sur quelques crustacés amphipodes provenant d'un dragage de l'Hirondelle au large de Lorient. Bulletin de la Société Zoologique de France 13: 39-42.

Dallwitz, M.J., T.A. Paine \& E.J. Zurcher, 1993 onwards. User's Guide to the DELTA System: A General System for Processing Taxonomic Descriptions. 4th edition. http://biodiversity.uno.edu/delta/

Dallwitz, M.J., T.A. Paine \& E.J. Zurcher, 1998. Interactive keys. In Information Technology, Plant Pathology and Biodiversity, ed. P. Bridge, P. Jeffries, D.R. Morse and P.R. Scott, pp. 201212. Wallingford: CAB International.

De Broyer, C., \& K. Jazdzewski, 1993. Contribution to the marine biodiversity inventory. A checklist of the Amphipoda (Crustacea) of the Southern Ocean. Documents de Travail de l'Institut Royal des Sciences Naturelles de Belgique 73: 1-154.

Della Valle, A., 1893. Gammarini del Golfo di Napoli. Fauna und Flora des Golfes von Neapel und der angrenzenden Meeresabschnitte 20: 1-948, pls 1-61.

Griffiths, C.L., 1977. Deep-sea amphipods from west of Cape Point, South Africa. Annals of the South African Museum 73(4): 93-104.

Gurjanova, E.F., 1962. [Amphipods of the northern part of the Pacific Ocean (Amphipoda-Gammaridea). Part 1]. Akademiya Nauk SSSR, Opredeliteli po Faune SSSR 74: 1-440. (In Russian).

Imbach, M.C., 1967. Gammaridean Amphipoda from the South China Sea. Naga Report 4(1): 39-167.

Lowry, J.K., 2000. Taxonomic status of amphipod crustaceans in the South China Sea with a checklist of known species. The Raffles Bulletin of Zoology, Supplement 8: 309-342.

Lowry, J.K., \& S. Bullock, 1976. Catalogue of the marine gammaridean Amphipoda of the Southern Ocean. Bulletin of the Royal Society of New Zealand 16: 1-187.

Lowry, J.K., \& H.E. Stoddart, 2002. First records of lysianassoid amphipods (Crustacea) from the Andaman Sea. In Biodiversity of Crustacea of the Andaman Sea. Proceedings of the International Workshop on the Crustacea in the Andaman Sea, Phuket Marine Biological Center, 29 November-20 December 1998, ed. N.L. Bruce, M. Berggren \& S. Bussawarit. Phuket Marine Biological Center Special Publication 23(1): 165-188.

Mills, E.L., 1972. T.R.R. Stebbing, the "Challenger" and knowledge of deep-sea Amphipoda. Proceedings of the Royal Society of Edinburgh (B) 72: 69-87.

Nayar, K.N., 1959. The Amphipoda of the Madras coast. Bulletin of the Madras Government Museum, New Series, Natural History Section 6(3): 1-59.
Nayar, K.N., 1966. On the gammaridean Amphipoda of the Gulf of Mannar, with special reference to those of the pearl and chank beds. Proceedings of the Symposium on Crustacea held at Ernakulam from January 12 to 15, 1965. Marine Biological Association of India 1: 133-168.

Poore, A.G.B., \& J.K. Lowry, 1997. New ampithoid amphipods from Port Jackson, New South Wales, Australia (Crustacea: Amphipoda: Ampithoidae). Invertebrate Taxonomy 11: 897-941.

Ren, X., \& L. Huang, 1991. [Studies on Gammaridea and Caprellidea (Crustacea: Amphipoda) from the northwest waters of the Antarctic Peninsula]. Studia Marina Sinica 32: 187323. [in Chinese and English]

Ruffo, S., \& U. Schiecke, 1977. Le specie Mediterranee del genere Lepidepecreum Bate \& Westwood (Amphipoda, Lysianassidae). Bollettino del Museo civico di Storia naturale, Verona 4: 429-447.

Shoemaker, C.R., 1942. Amphipod crustaceans collected on the Presidential Cruise of 1938. Smithsonian Miscellaneous Collections 101(11): 1-52.

Stebbing, T.R.R., 1888. Report on the Amphipoda collected by H.M.S. Challenger during the years 1873-1876. Report on the Scientific Results of the Voyage of H.M.S. Challenger during the years 1873-76, Zoology, 29: 1-1737, pls. 1-210.

Stebbing, T.R.R., 1906. Amphipoda. I. Gammaridea. Das Tierreich 21: 1-806.

Stimpson, W., 1864. Descriptions of new species of marine Invertebrata from Puget Sound, collected by the naturalists of the North-west Boundary Commission, A.H. Campbell, Esq., Commissioner. Proceedings of the Academy of Natural Sciences of Philadelphia 1864: 153-161.

Surya Rao, K.V., 1972. Intertidal amphipods from the Indian Coast. Proceedings of the Indian National Science Academy 38(3-4): 190-205.

Thurston, M.H., \& E. Allen, 1969. Type material of the families Lysianassidae, Stegocephalidae, Ampeliscidae and Haustoriidae (Crustacea: Amphipoda) in the collections of the British Museum (Natural History). Bulletin of the British Museum (Natural History) (Zoology) 17: 347-388.

Vinogradov, G.M., 1994. [Amphipods (Crustacea) from seep fields and nearby common benthic communities of the northern and eastern Pacific]. Trudy Instituta Okeanologii Akademii Nauk SSSR 131: 100-125. (In Russian with English summary).

Manuscript received 2 February 2000, revised 7 July 2000 and accepted 6 September 2000.

Associate Editor: D.J. Bickel.

\section{Index to species}

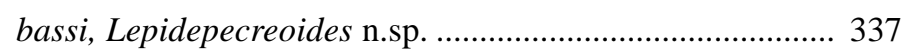

baudini, Lepidepecreum n.sp. .......................................... 347

chincui, Lepidepecreoides n.sp. ......................................... 340

dampieri, Lepidepecreum n.sp. ........................................ 351

flindersi, Lepidepecreum n.sp. ......................................... 351

foraminiferum, Lepidepecreum Stebbing ........................... 354

freycineti, Lepidepecreum n.sp......................................... 357

infissum, Lepidepecreum Andres ....................................... 360

nubifer, Lepidepecreoides J.L. Barnard ............................. 343

talboti, Lepidepecreoides n.sp.......................................... 343

torresi, Lepidepecreoides n.sp........................................... 344

tourville, Lepidepecreum n.sp. ......................................... 360

urometacarinatum, Lepidepecreum Andres ........................ 363

xenopus, Lepidepecreoides K.H. Barnard ............................ 344 